\title{
Downsizing by Shutdown in Red Galaxies
}

\author{
A. Cattaneo ${ }^{1 \star}$, A. Dekel ${ }^{2, \dagger}$, S. M. Faber ${ }^{3}$, B. Guiderdoni ${ }^{4}$ \\ ${ }^{1}$ Astrophysikalisches Institut Potsdam, an der Sternwarte 16, 14482 Potsdam, Germany \\ ${ }^{2}$ Racah Institute of Physics, Hebrew University of Jerusalem, 91904 Jerusalem, Israel \\ ${ }^{3}$ UCO/Lick Observatory, University of California, USA \\ ${ }^{4}$ Centre de Recherche Astrophysique de Lyon, France \\ `acattaneo@aip.de, ${ }^{\dagger}$ dekel@phys.huji.ac.il
}

3 November 2018

\begin{abstract}
We address the origin of the 'downsizing' of elliptical galaxies, according to which the stars in more massive galaxies formed earlier and over a shorter period than those in less massive galaxies. We show that this could be the natural result of a shutdown of star formation in dark matter haloes above a critical mass of $\sim 10^{12} M_{\odot}$. This is demonstrated using a semi-analytic simulation of galaxy formation within the standard hierarchical scenario of structure formation. The assumed threshold mass is motivated by the prediction of stable shock heating above this mass and the finding that such a shutdown reproduces the observed distribution of galaxies in luminosity and colour. The shutdown at a critical halo mass introduces a characteristic stellar mass for the transition of galaxies into the 'red sequence' of the galaxy colour-magnitude diagram. Central galaxies of haloes that are more massive today have reached this mass earlier and can therefore grow further along the red sequence by dry mergers, ending up more massive and containing older stars. Small galaxies formed in haloes below the critical mass can shutdown late, when they fall into haloes above the critical mass and become satellites. While our semi-analytic simulation that incorporates an explicit shutdown reproduces downsizing as inferred from the stellar ages of ellipticals, we explain why it is much harder to detect downsizing using the mass functions of different galaxy types.
\end{abstract}

Key words: galaxies: clusters — galaxies: ellipticals — galaxies: evolution — galaxies: formation - galaxies: haloes

\section{INTRODUCTION}

The term 'downsizing' was coined by Cowie et al. (1996) to describe the decline with time of the $K$-band restframe luminosity of the galaxies with the highest specific star formation rate $\dot{M}_{\text {star }} / M_{\text {star }}$ (SFR) as observed in the redshift interval $0.2<z<1.7$. This 'downsizing in time' has been confirmed by later studies Guzman et al. 1997; Brinchmann \& Ellis 2000; Kodama et al. 2004; Juneau et al. 2005; Bell et al. 2005; Noeske et al. 2007). It can be viewed as part of a more general phenomenon valid robustly across the Hubble sequence of galaxy types, in the sense that galaxies of later type, which are typically less massive, are known to have formed their stars most efficiently at later times and over longer periods (Searle et al. 1973; Tinsley 1973; Sandage 1986). A possibly related downsizing is evident in the accretion histories of supermassive black holes (Steffen et al.
2003; Ueda et al. 2003; Heckman et al. 2004; Barger et al. 2005; Hasinger et al. 2005).

Another form of downsizing, termed 'archaeological downsizing' (Thomas et al. 2005), is inferred from the stellar populations of today's galaxies. Star formation histories derived from observed line indices and abundance ratios using stellar evolution models reveal a strong correlation between mean stellar age and galactic stellar mass both in elliptical galaxies (Nelan et al. 2005; Thomas et al. 2005; Graves et al. 2007) and in the general population of galaxies from the large Sloan Digital Sky Survey (SDSS; Heavens et al. 2004; Jimenez et al. 2005; Panter et al. 2006). In the current study we focus on archaeological downsizing, as we address red galaxies that show only little active star formation at present.

At a first glance, the observed downsizing seems to be in conflict with the standard wisdom of hierarchical structure formation, according to which smaller objects collapse ear- 
lier and gradually assemble into more massive objects. This apparent conflict is relaxed when realizing that star formation and gravitational assembly are two distinct processes. In particular, stars can form first in the small building blocks of today's massive galaxies without violating the assembly hierarchy of each galaxy. In fact, if gas processes limit galaxy formation to dark matter haloes above a minimum mass, a certain downsizing trend arises naturally from the hierarchical dark matter assembly process itself Neistein et al. 2006). Still, the origin of downsizing as observed requires a more quantitative theoretical understanding.

Recent developments in the modelling of galaxy formation (e.g. Cattaneo et al. 2006; Bower et al. 2006; Croton et al. 2006) have been driven by the realization that galaxies are divided into two major distinct types: blue star-forming late-type galaxies in low-density environments, red and dead early-type galaxies in groups and clusters (Lin et al. 1997, 1999; Im et al. 2001; Strateva et al. 2001; Kauffmann et al. 2003; Baldry et al. 2004; Balogh et al. 2004; Bell et al. 2004; Hogg et al. 2004; Weiner et al. 2005; see Dekel \& Birnboim 2006 for a summary of the bimodality phenomenon). Combined with the fact that blue galaxies are confined below a critical stellar mass of $M_{\text {star }}^{\text {crit }} \sim 3 \times 10^{10} M_{\odot}$ while the population above this mass is dominated by red galaxies, the colour bimodality itself is evidence for downsizing. It indicates that the most massive galaxies, the red ones, have converted their gas into stars several billion years ago, while less massive galaxies, which are mostly blue, are still making stars.

Downsizing is not only a reflection of the galaxy bimodality. It is also valid separately within each of the two populations, the red one (Nelan et al. 2005; Thomas et al. 2005; Graves et al. 2007) and the blue one (Drorv et al. 2006; Noeske et al. 2007). Less massive galaxies contain younger stars even within the same spectral type. Nevertheless, we are tempted to consider the possibility that downsizing and bimodality originate from the same underlying physics.

The existence of a characteristic mass for massive galaxies emerges from the the competition between the gravitational dynamical time and the radiative cooling time (Rees \& Ostriker 1977; Binnev 1977; Silk 1977; White \& Rees 1978; Blumenthal et al. 1984). Birnboim \& Dekel (2003) and Dekel \& Birnboim (2006) showed that a stable shock can expand to the virial radius and be supported against gravity only in haloes above a critical mass of $M_{\text {shock }} \sim 10^{12} M_{\odot}$, producing a hot medium at the virial temperature of $T \geqslant 10^{6} \mathrm{~K}$. In smaller haloes, efficient cooling does not allow an extended stable shock, so the accreting gas flows cold into the centre, leading to the buildup of discs and efficient star formation. This phenomenon was detected in parallel in cosmological simulations (Kereš et al. 2005; Birnboim et al. 2007; Cattaneo et al. 2007), confirming the existence of a threshold mass that is quite independent of redshift. Dekel \& Birnboim (2006) argued that the transition from cold flows to the shutdown of gas supply and star formation at $M_{\text {shock}}$, which is predicted to be more pronounced after $z \sim 2-3$, naturally leads to many of the observed features associated with the galaxy bimodality. In particular, the bimodality scale $M_{\text {star }}^{\text {crit }}$ is the typical stellar mass of the central galaxy in a halo with mass $M_{\text {shock }}$. The im- plications of such a scenario have been studied using semianalytic simulations by Cattaneo et al. (2006), Croton et al. (2006) and Bower et al. (2006), each applying a slightly different shutdown procedure driven by halo mass (plus, sometimes, bulge mass or central black hole mass). The formation of the red sequence through the quenching and reddening of blue galaxies has also been studied by Bell et al. (2004) and Faber et al. (2007). Here we highlight a particular aspect of the quenching mechanism, associated with the fact that the critical mass for quenching is roughly constant after $z \sim 2-3$.

In this article we demonstrate that the shutdown of star formation above a critical halo mass $M_{\text {crit }} \sim M_{\text {shock }}$ at $z<3$ accounts for downsizing in the red galaxy population. The key idea is that central galaxies stop making stars and enter the red sequence at a critical stellar mass $M_{\text {star }}^{\text {crit }}$ of the order of the baryonic mass in a halo of mass $M_{\text {shock }}$. Galaxies with higher final stellar mass reach $M_{\text {star }}^{\text {crit }}$ earlier and therefore have more time to grow through dissipationless ('dry') mergers along the red sequence.

There are three complications to this simple picture, which we discuss in this article. First, satellite galaxies shut down earlier than central galaxies with the same mass, and they often do it before they have reached $M_{\text {star }}^{\text {crit }}$ (e.g. because their halo has merged into another one with mass $>M_{\text {shock}}$ ). Second, the expected penetration of narrow cold streams through the hot media of massive high-redshift haloes elevates the effective $M_{\text {crit }}$ to values above $M_{\text {shock }}$ at $z \geqslant 3$ (Dekel \& Birnboim 2006; Cattaneo et al. 2006). Third, additional shutdown mechanisms may be at work, e.g. the exhaustion of gas after major mergers and/or feedback from the central black hole. We have attempted to mimick these effects by intoducing a further shutdown criterion related to the bulge-to-disc ratio. The inclusion of a bulge-to-disc ratio criterion as a sufficient condition for quenching in addition to the halo-mass criterion improves the fit to the joint colour-magnitude distribution of galaxies by accounting for the presence of red galaxies in haloes below $M_{\text {crit }}$, but its overall effect is fairly minor (Cattaneo et al. 2006, Fig. 9).

Downsizing has also been involved to explain the way the stellar-mass functions of galaxies of different colors or morphological types evolve with time. In particular, while the comoving number density of blue galaxies has not changed much since $z \sim 1$, the number of red galaxies has seemingly grown by a larger factor at the faint end than at the bright end (Rudnick et al. 2003; Bell et al. 2004; Drory et al. 2004; Bundy et al. 2005; Drory et al. 2005; Borch et al. 2006; Pannella et al. 2006; Faber et al. 2007). We argue below that the interpretation of this behavior as downsizing is hampered by severe measurement uncertainties (see the Appendix) and conceptual misunderstandings.

The paper outline is as follows. In $\S 2$, we describe our model of galaxy formation explored by means of a semianalytic modelling technique applied to merger trees from a cosmological N-body simulation, following Cattaneo et al. (2006). In $\S 3$, we elaborate on how the shutdown of star formation leads to downsizing. In $\S 4$, we show how the shutdown produces downsizing in the buildup of the red sequence, in the sense that the high-mass end is populated at earlier times. In $\S 5$, we perform a comparison with the archeological downsizing inferred from the stellar ages in elliptical galaxies (Thomas et al. 2005). In $\S 6$, we consider 
the characteristic times of galaxy formation in terms of star formation history and mass assembly history, and use this analysis to address the apparent conflict between downsizing and the hierarchical model. In $\S 7$, we explain how downsizing in star formation and upsizing in mass assembly combine to produce the evolution of the mass functions of red and blue galaxies, and why it is hard to detect downsizing using the mass functions of different galaxy types. In $\S 8$, we summarize the conclusions of the article. The downsizing in blue galaxies will be addressed in a separate publication.

\section{THE GALAXY FORMATION MODEL}

GalICS (Galaxies In Cosmological Simulations; Hatton et al. 2003) is a method to simulate the formation of galaxies in a $\Lambda$ CDM Universe. It combines high-resolution cosmological N-body simulations of the gravitational clustering of the dark matter with a semi-analytic (SAM) approach to the physics of the baryons (gas accretion, galaxy mergers, star formation and feedback). The version of GalICS used here, as well as the adopted values of the parameters, are the same as described in Cattaneo et al. (2006, and references therein). We summarize here the relevant issues.

\subsection{Dark-matter simulation}

The cosmological N-body simulation that follows the hierarchical clustering of the dark-matter component has been carried out with the parallel tree code. The assumed cosmological model is a flat $\Lambda$ CDM Universe with a cosmological constant of $\Omega_{\Lambda}=0.667$, a Hubble constant of $H_{0}=66.7 \mathrm{~km} \mathrm{~s}^{-1}$, and a $\Lambda$ CDM power spectrum of initial fluctuations normalized to $\sigma_{8}=0.88$. The computational volume is a cube of side $(150 \mathrm{Mpc})^{3}$ with $256^{3}$ particles of $8.3 \times 10^{9} \mathrm{M}_{\odot}$ each and a smoothing length of $29.3 \mathrm{kpc}$. The simulation produced 100 output snapshots spaced logarithmically in the expansion factor $(1+z)^{-1}$ from $z=35.59$ to $z=0$.

We have analysed each snapshot with a friend-of-friend algorithm (Davis et al. 1985) to identify virialized haloes containing more than 20 particles. The minimum halo mass is thus $1.65 \times 10^{11} M_{\odot}$. The three global properties characterizing each halo to be used in the SAM are the virial mass, the virial density, and the spin parameter. Merger trees are constructed by linking the haloes identified in each snapshot with their progenitors in the previous snapshot, that is, all predecessors from which the halo has inherited one or more particles. We do not use substructure information from the N-body simulation. Instead, once a halo becomes a subhalo of another halo, we switch from following its evolution with the N-body integrator to an approximate treatment based on semi-analytic prescriptions.

\subsection{Semi-analytic modeling of gas processes}

A newly identified halo is assigned a gas mass based on a universal baryon fraction $\Omega_{\mathrm{b}} / \Omega_{0}=0.135$. The conditions for the formation of a galaxy at its centre are that the halo is gravitationally bound and that its angular momentum parameter is $\lambda<0.5$. We have simulated a 'standard' and a 'new' model, which differ in the way gas from the halo accretes onto the central galaxy.

In the 'standard' model, the initial gas distribution is a singular isothermal sphere, at the virial temperature, truncated at the virial radius. The cooling time of the hot gas is computed using the radiative cooling function of Sutherland \& Dopita (1993). The gas for which both the cooling time and the free fall time are shorter than the timestep $\Delta t$ between two $\mathrm{N}$-body snapshots is accreted onto the central galaxy during that time interval. The transfer of baryons from the halo to the galaxy is accompanied by an inflow of hot gas to keep the hot gas distribution a truncated singular isothermal sphere. The numerical accuracy and the self-consistency of this cooling algorithm have been verified by comparison with a cosmological hydrodynamic simulation based on the same physics and the same dark-matter realization (Cattaneo et al. 2007).

In the 'new' model, we introduce the proposed quenching above a threshold halo mass, following Dekel \& Birnboim (2006). Once a halo grows above $M_{\text {shock }} \sim 10^{12} M_{\odot}$, the halo gas is assumed to be shockheated to the virial temperature. Unlike previous models, once heated, the hot gas is kept hot forever. This can be due to several different long-term quenching mechanisms, such as self-regulated radio-AGN feedback (e.g. Croton et al. 2006; Cattaneo \& Teyssier 2007, and references therein), shocked accretion (Birnboim et al. 2007), or gravitational quenching by clumpy accretion (Dekel \& Birnboim 2007; Khochfar \& Ostriker 2007). The critical mass for quenching is assumed to be

$M_{\text {crit }}=M_{\text {shock }} \times \max \left\{1,10^{1.3\left(z-z_{\mathrm{c}}\right)}\right\}$,

where $M_{\text {shock }} \sim 10^{12} M_{\odot}$ is the shock-heating scale and the term $\propto 10^{1.3 z}$ accounts for the penetration of cold streams in massive haloes at high redshift (Dekel \& Birnboim 2006, Fig. 7). In Cattaneo et al. (2006), we set the parameters to $M_{\text {shock }}=2 \times 10^{12} M_{\odot}$ and $z_{\mathrm{c}}=3.2$ by optimizing the way the new model fits the colour-magnitude distribution at $z \simeq 0$ and the Lyman-break galaxy luminosity function at $z \simeq 3$.

In the standard model, there is no explicit shutdown of cooling, and the mass and luminosity of the central galaxy grow roughly linearly with halo mass over the entire range resolved by the N-body simulation. Shutting down the hot accretion mode when $M_{\text {halo }}>M_{\text {crit }}$ introduces a characteristic mass (Fig. 1) and luminosity, which mark the separation between blue and red galaxies.

Initially, all galaxies are assumed to form at a distance $r_{i}=0$ from the centre of their halo. When two or more haloes merge, their galaxies are repositioned at a distance $r_{f}$ from the centre of the new halo, determined such that $r_{f} \rightarrow r_{i}$ for $M_{f} / M_{i} \rightarrow 1$ and $r_{f} \rightarrow r_{\text {vir }}$ for $M_{f} / M_{i} \rightarrow \infty$ (where $M_{i}$ and $M_{f}$ are the halo mass before and after the merger and $r_{\text {vir }}$ is the virial radius of the merged halo. Halo mergers rapidly create haloes with more than one galaxy, which we identify as galaxy groups and clusters. However, only the central galaxy is allowed to accrete gas from the halo (prior to quenching).

\subsection{Morphologies}

GalICS models a galaxy with three components: a disc, a bulge and a transitional 'starburst' component. Each com- 

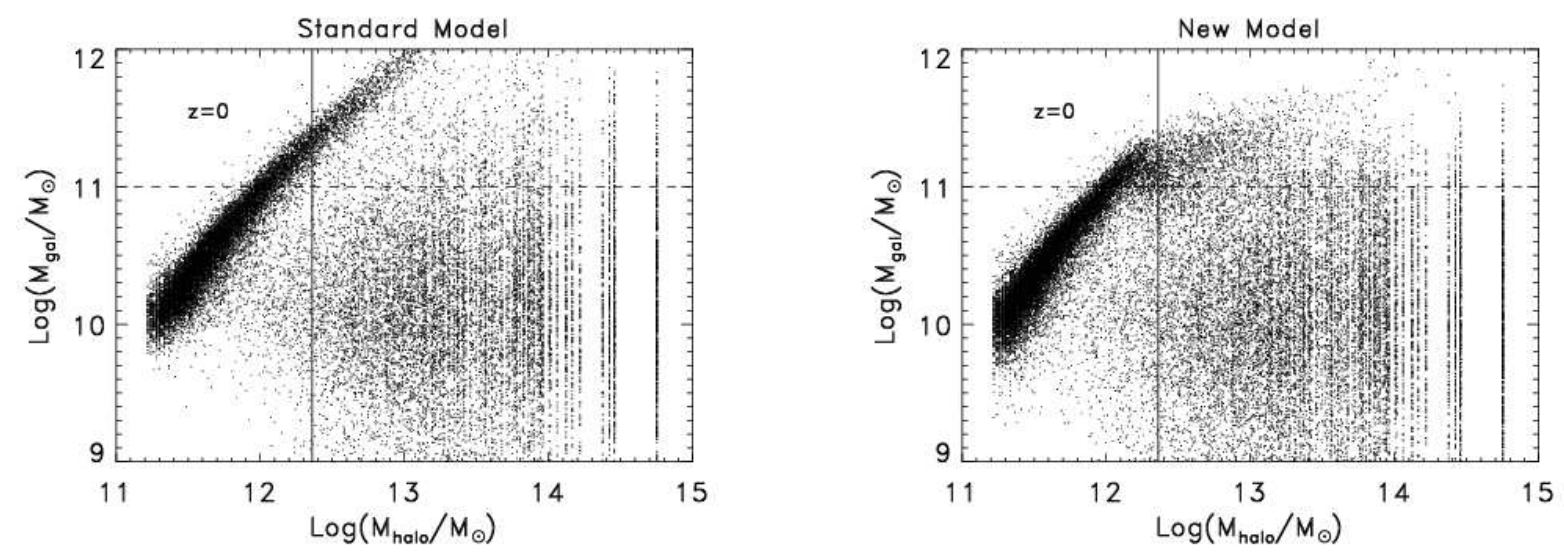

Figure 1. Galaxy stellar mass versus halo mass in the standard model (left) and the new model with shutdown (right). In the new model, the general growth of $M_{\text {star }}$ with $M_{\text {halo }}$ breaks off at $M_{\text {halo }} \sim M_{\text {shock }} \sim 2 \times 10^{12} M_{\odot}$. Differences at $M_{\text {halo }}<M_{\text {shock }}$ are due to the additional shutdown of accretion onto the galaxies that have become bulge-dominated. Objects with high $M_{\text {halo }}$ but low $M_{\text {gal }}$ are mostly satellites that have fallen into larger haloes.

ponent may contain stars and cold gas, while the hot gas is treated as a component of the halo. Only the disc of the central galaxy accretes gas from the halo. The bulge grows by mergers and by disc instabilities. While stars are trasferred directly from the disc to the bulge, the gas in transit from the disc to the bulge is assumed to pass through an intermediate starburst component, where its star formation timescale decreases by a factor of ten with respect to that of the bulge (Section 2.4). Stars formed in the starburst are moved to the bulge after they have reached an age of $100 \mathrm{Myr}$. The only gas in the bulge is that from stellar mass loss $(\S 2.4)$.

The disc profile is assumed to be exponential, with its radius determined by conservation of angular momentum. The bulge is assumed to have a Hernquist (1990) profile and its radius is determined based on an energy conservation argument.

We now elaborate on the two mechanisms for transferring baryons from the disc to the spheroid. First, disc instabilities transfer matter from the disc to the spheroid (stars to the bulge, gas to the starburst) until the bulge is massive enough to stabilize the disc. The stability criterion is $v_{\text {rot }}<0.7 v_{\mathrm{c}}$, where $v_{\text {rot }}$ and $v_{\mathrm{c}}$ are the disc's rotation and circular velocity at the disc's half mass radius van den Bosch 1998). Second, dynamical friction, modelled as in Hatton et al. (2003), drives galaxies to the centre of their dark matter halo and is the dominant cause of galaxy merging, although we also include satellite-satellite encounters. The fraction of the disc that is transferred to the bulge in a merger grows with the mass ratio of the merging galaxies. It ranges from zero for a very minor merger to unity for an equal mass merger. This simplified picture of the dynamics of morphological transformations provides results consistent with the key observational constraints such as the Faber-Jackson relation and the Fundamental Plane of spheroids (Hatton et al. 2003).

\subsection{Star formation and feedback}

The star formation law is the same for all components:
$\dot{M}_{\text {star }}=\frac{M_{\text {cold }}}{\beta_{*} t_{\text {dyn }}}(1+z)^{\alpha_{*}}$.

The mass of cold gas, $M_{\text {cold }}$, refers to the component in question, and $t_{\mathrm{dyn}}$ is the dynamical time (corresponding to half a rotation for discs and half a crossing time for bulges). In the standard GalICS model, star formation is activated when the gas surface density is $\Sigma_{\text {gas }}>20 m_{\mathrm{p}} \mathrm{cm}^{-2}\left(m_{\mathrm{p}}\right.$ is the proton mass). Furthermore, by imposing a minimum halo mass of $M_{\text {halo }}=1.65 \times 10^{11} M_{\odot}$ due to the N-body resolution, we effectively assume that there is no star formation in haloes below this mass, potentially mimicking the effects of more aggressive supernova feedback in small haloes (see below). Moreover, in the new model we assume a complete shutdown of star formation in haloes with mass $>M_{\text {crit }}$ by removing all the cold present in any component of these galaxies and by moving this gas to the halo's hot component.

The star formation efficiency parameter is assumed to be $\beta_{*}=50$ (Guiderdoni et al. 1998), the same for all components. We have $\alpha_{*}=0$ in the standard GalICS model but allow an enhanced star-formation rate at high redshift by adopting $\alpha_{*}=0.6$ in the new model, to mimic the rapid accretion by cold flows. This improves the fit to the luminosity function of Lyman-break galaxies (Cattaneo et al. 2006).

Gas in transit from the disc to the bulge, whether by mergers or disc instabilities, passes through a starburst phase in which the SFR grows by a factor of 10 based on the dynamical time of the bulge. This high SFR is obtained by assuming that the starburst radius is ten times smaller than the final bulge radius (see the hydrodynamic simulation in Cattaneo et al. 2005). As we anticipated when we described the different components of a galaxy, the stars formed in the starburst component stay in the starburst component for $100 \mathrm{Myr}$ and then are moved to the bulge. The properties of low redshift galaxies are insensitive to the starburst star formation timescale as long as it is much shorter than the Hubble time.

We assume a Kennicutt (1983) initial mass function. Stars are evolved between snapshots using substeps of at most 1 Myr. During each sub-step, stars release mass and energy into the interstellar medium. Most of the mass comes from the red giant and the asymptotic giant branches of 
Standard Model

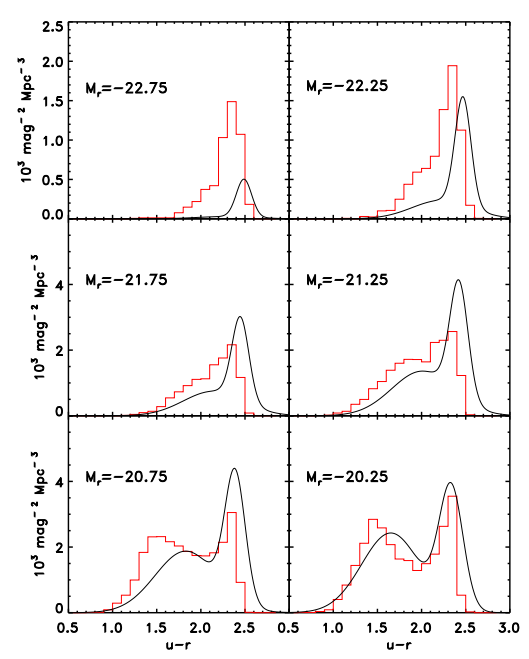

No Additional Bulge Mass Criterion

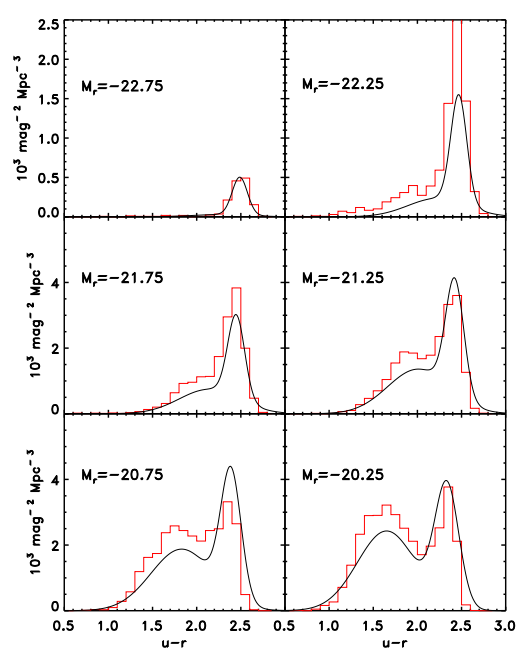

New Model

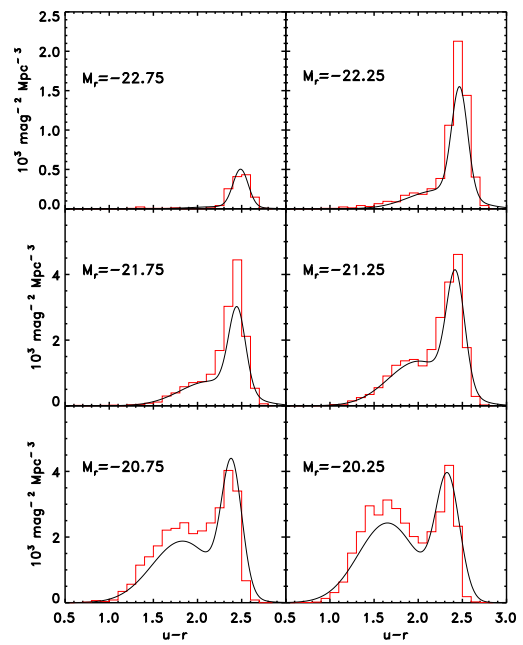

Figure 2. The the $u-r$ colour distribution in $r$-band magnitude bins for the SDSS data (Baldrv et al. 2004; black smoothed histogram) and for three different versions of our SAM (red curves). Left: the standard model without an explicit shutdown of central galaxies but with a standard shutdown of gas accretion onto satellites. Middle: A model with shutdown of star formation once $M_{\text {halo }}>M_{\text {crit }}$. Right: Same as in the middle, with the additional shutdown when $M_{\text {bulge }}>M_{\text {star }} / 2$ (reproduced from Cattaneo et al. 2006).

stellar evolution, while most of the energy comes from shocks due to supernova explosions. The enriched material released in the late stages of stellar evolution is mixed with the cold phase, while the energy released from supernovae is used to reheat the cold gas and to return it to the hot phase in the halo. Reheated gas is ejected from the halo if the potential is shallow enough. The rate of mass loss through supernovadriven winds $\dot{M}_{\mathrm{w}}$ is determined by the equation

$\frac{1}{2} \dot{M}_{\mathrm{w}} v_{\mathrm{esc}}^{2}=\epsilon_{\mathrm{SN}} \eta_{\mathrm{SN}} E_{\mathrm{SN}} \dot{M}_{\mathrm{star}}$

where $E_{\mathrm{SN}}=10^{51} \mathrm{erg}$ is the energy of a supernova, $\eta_{\mathrm{SN}}=$ 0.0093 is the number of supernovae for $1 M_{\odot}$ of stars formed and $v_{\text {esc }}$ is the escape velocity (Dekel \& Silk 1986). In GalICS, we use $v_{\text {esc }} \simeq 1.84 v_{\mathrm{c}}$ for discs and $v_{\text {esc }}=2 \sigma$ for bulges and starbursts. The supernova efficiency $\epsilon_{\mathrm{SN}} \simeq 0.2$ is similar to those commonly adopted in SAMs (Somerville \& Primack 1999; Cole et al. 2000).

\subsection{Stardust}

The STARDUST model (Devriendt et al. 1999) provides the spectrum of a stellar population as a function of age and metallicity and includes a phenomenological treatment of the reprocessing of stellar light by dust. GalICS uses STARDUST to compute the spectrum of each component and to output galaxy magnitudes.

Dust absorption is computed with a phenomenological extinction law that depends on the column density of neutral hydrogen, the metallicity of the obscuring material and the randomly selected inclination angle (for spirals). The reemitted spectrum is the sum of four templates (big and small carbon grains, silicates, and polycyclic aromatic hydrocarbons). Their relative weights are chosen to reproduce the relation between bolometric luminosity and infrared colours observed locally in IRAS galaxies. In GalICS we only consider each component's self-absorption, e.g. the dust in a starburst only obscures the starburst's light, not the old bulge stellar population.

\section{SHUTDOWN AND DOWNSIZING}

In the new model, the accretion of gas onto the central galaxy is shut down once $M_{\text {halo }}>M_{\text {crit }}$. Both in the standard and the new model, as common in other SAMs, the accretion of gas onto satellite galaxies is not allowed 1 Figure 1 shows that the stellar mass keeps pace with the halo mass up to $M_{\text {crit }}$ and then breaks off in the new model. It also shows the effect of preventing the accretion of gas onto satellite galaxies, which are shown by the point clouds in the lower right part of the diagrams.

In order to maximize the quenching effect, whenever the shutdown criteria are satisfied we actually shut down star formation altogether, that is, in addition to having the halo gas shock heated and to keeping it hot, we assume that the cold gas is removed from the galaxies and that it is added to the hot halo. This can be achieved in central galaxies by thermal evaporation (Nipoti \& Binnev 2007), and in satellite galaxies by ram-pressure stripping (e.g. Bahcall 1977; Gallagher 1978). Cattaneo et al. (2006) demonstrated that this robust shutdown leads to a good fit to the observed

1 Admittedly, this procedure may be too strict and should be revised, especially in small haloes with no hot medium, where anyway it may be hard to distinguish between central galaxies and satellites (e.g. Cattaneo et al. 2007; also see McCarthy et al. 2007). 


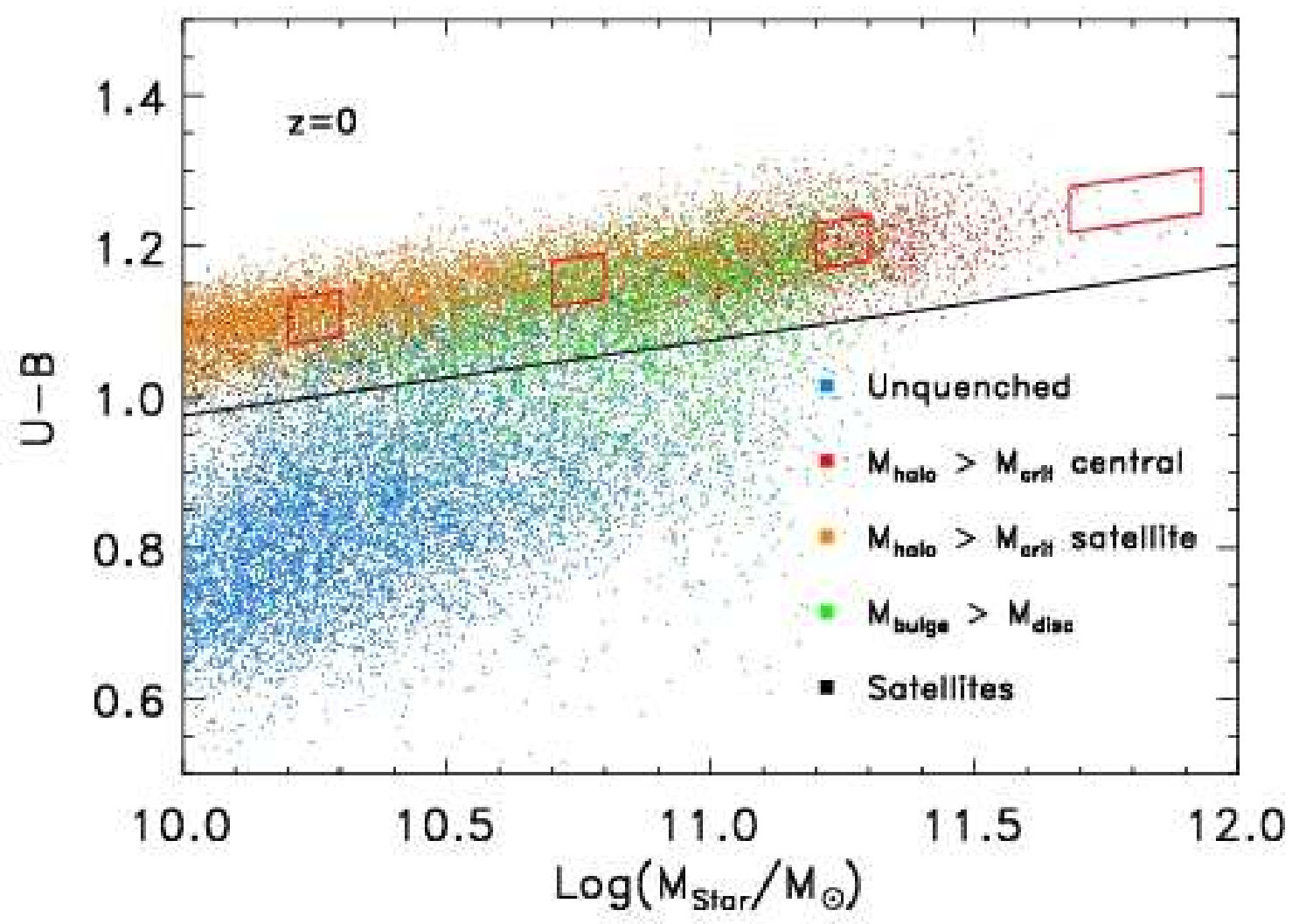

Figure 3. Colour-mass diagram for the new model with shutdown. The 'green valley' separating the red sequence from the blue cloud is marked by the black line. Blue symbols mark galaxies in which the SFR has not been explicitly shut down. Red symbols denote central galaxies in which the SFR has been shut down because their halo has grown above the critical mass. Orange symbols show the population of satellite galaxies in haloes above the critical mass. Green symbols are central galaxies of haloes with $M_{\text {halo }}<M_{\text {crit }}$, where the formation of stars has been shut down because the bulge has become the dominant component. Black symbols refer to galaxies that do not accrete gas simply because they are satellites. In this diagram galaxies have been coloured according to their status at $z=0$, and not according to the first mechanism that has intervened and caused the shutdown of star formation in the history of a given galaxy. The red parallelograms show the four bins along the red sequence from which we have selected the galaxies for Figs. 4 and 5

colour-magnitude distribution (Fig. 2] centre). In the absence of such an explicit shutdown the standard model predicts too many luminous and blue galaxies (Fig. 2 left).

The only slight disagreement between the predictions of the simple model of shutdown by halo mass (red curves in the panel marked "no additional bulge mass criterion') and the SDSS data (black smoothed histograms) is a small excess of blue galaxies at $-22.25 \lesssim M_{r}$, which arises from the absence of quenched central galaxies with $M_{\text {star }} \lesssim 1-2 \times 10^{11}$, since their haloes are below the shock-heating scale. This is the range where the elliptical population is dominated by rather discy configurations, which do not show X-ray emission in excess of the contribution of discrete sources (Bender et al. 1989). These galaxies could have been possibly quenched by another mechanism, such as quasar feedback after gasrich mergers (Springel et al. 2005a; Hopkins et al. 2007), following the notion that discy ellipticals emerge from wet mergers (e.g. Cox et al. 2006), which also trigger quasar activity (Toomre \& Toomre 1972; Cattaneo et al. 1999; Kauffmann \& Haehnelt 2000; Cattaneo et al. 2005a,b;
Springel et al. 2005b; Hopkins et al. 2005). We do not attempt here to model quasar feedback in detail. We simply mimic its effect by stopping the accretion onto a galaxy whenever it is dominated by its bulge component, even if it is still in a halo with $M_{\text {halo }}<M_{\text {crit }}$. This can occur only after a major merger or a sequence of minor mergers since the disc instability criterion does not permit the growth of bulges with $M_{\text {bulge }}>M_{\text {star }} / 2$ (see Section 2.3). This additional quenching criterion slightly improves the fit to the SDSS colour-magnitude distribution near $M_{r} \simeq-21.5$ (the 'new model' in Fig 2). Note that the cold gas is not removed when a galaxy is quenched by this criterion alone. While this is clearly a simplified ad hoc implementation of quasar feedback, it has negligible effects at the bright end and at the faint end, and it hardly affects the main results of our current analysis.

One could conceive an alternative scenario where quasar feedback after galaxy mergers is the main shutdown mechanism (e.g. Hopkins et al. 2007), although it would have a hard time explaining the long-term maintenance of quenching. There is some observational evidence for such a mecha- 


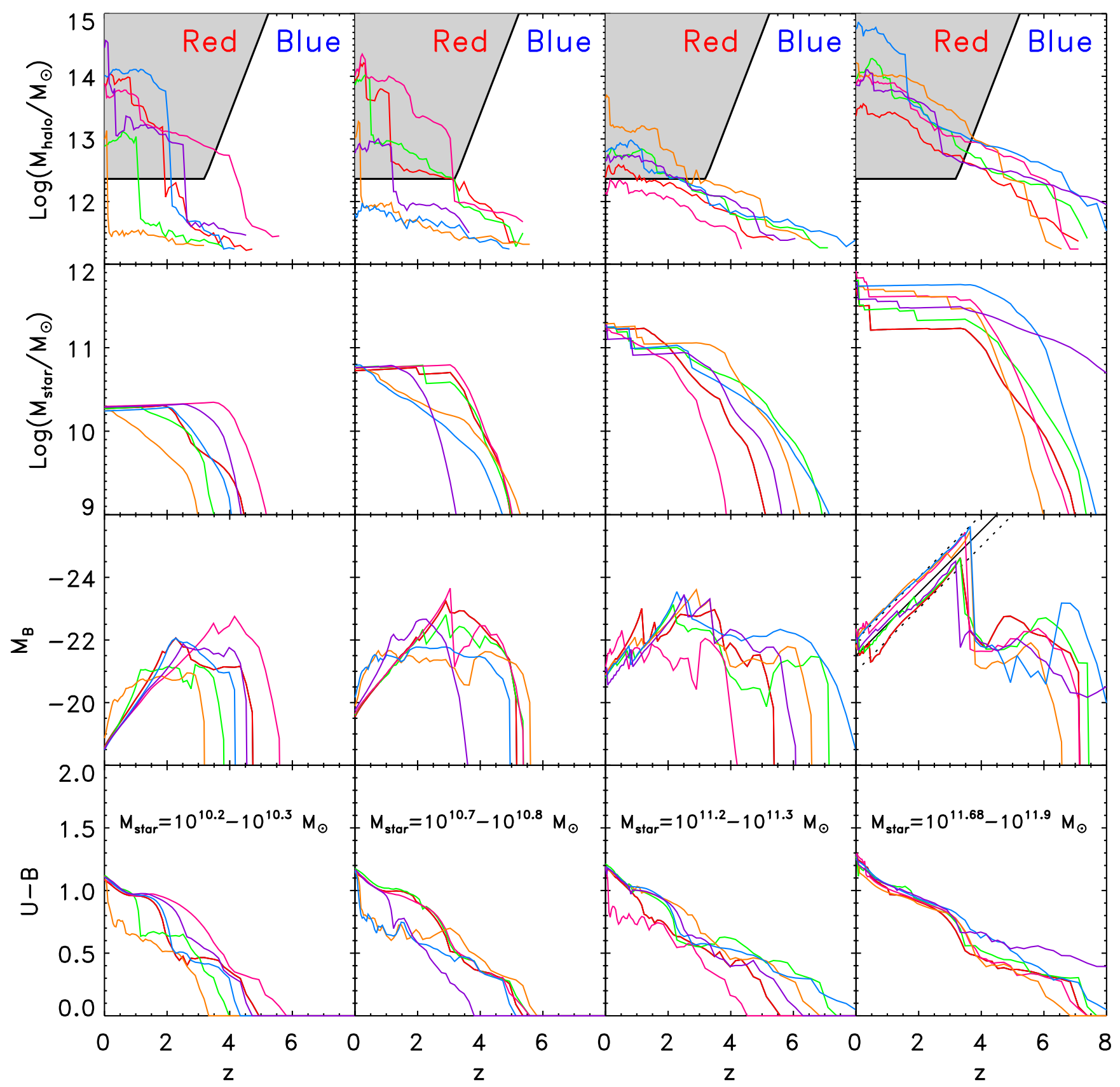

Figure 4. Growth of halo mass (top) and stellar mass (bottom) for 24 galaxies randomly sampled from the four mass bins indicated (see Fig. 31). The growth curves refer to the most massive progenitors. The bold black line in the upper panels is $M_{\text {crit }}(z)$ above which cooling and star formation are shut down (Eq. 1). After $z \sim 3, M_{\text {crit }} \sim M_{\text {shock }} \sim 2 \times 10^{12} M_{\odot}$. After $M_{\text {halo }}$ has crossed this threshold, the central galaxy ceases to form stars and passively turns red and dead. Growth of the stellar mass after this point occurs only via mergers. The sudden jumps in $M_{\text {halo }}$ occur when a galaxy becomes a satellite in a larger halo. The passive fading of giant ellipticals after $z \sim 3-4$ is well described by the simple relation $M_{B} \sim-21.5-z \pm 0.5$ (solid line between the two dotted lines corresponding to $\pm 0.5 \mathrm{mag})$ 
nism in action (Schawinski et al. 2007). Modelling this specific scenario is beyond the scope of the present paper, where we focus on the effect of halo quenching on downsizing.

In summary, the new model stops the accretion of gas onto a galaxy when at least one of the following three conditions is satisfied: (a) $M_{\text {halo }}>M_{\text {crit }}$, given by Eq. 1, or (b) $M_{\text {bulge }}>M_{\text {star }} / 2$, (c) the galaxy has become a satellite. When the quenching is by condition (a), the cold gas is explicitly removed, but even in the other cases the starved galaxies rapidly exhaust their gas and stop making stars. Fig. 3 illustrates via different colours the role of these three different mechanisms in producing the galaxy bimodality. We see that halo-mass quenching (a) is the main mechanism for $M_{\text {star }} \gtrsim 2 \times 10^{11} M_{\odot}$, and that the bulge quenching criterion (b) plays a role near $M_{\text {star }} \sim 10^{11} M_{\odot}$, while most low mass galaxies are both quenched by halo mass (a) and satellites (c). Only $3 \%$ of the red sequence at $M_{\text {star }}>10^{10} M_{\odot}$ is made of galaxies that are quenched only because they are satellites (c). Although $57 \%$ of the red sequence is composed of galaxies with $M_{\text {bulge }}>M_{\text {star }} / 2$, only $24 \%$ of red galaxies are quenched by criterion (b) only; $\sim 10 \%$ of the galaxies quenched by criterion (b) only are red because they are dusty. These galaxies are remnants of recent gas-rich mergers. They have ceased to accrete gas, but they have not yet ceased to make stars. They account for $\sim 2 \%$ of the red sequence at $M_{\text {star }}>10^{10} M_{\odot}$. About $8 \%$ of the red sequence at $M_{\text {star }}>10^{10} M_{\odot}$ is made of galaxies that have not been explicitly quenched by any of the three mechanisms above (blue points in Fig. 3). The breakdown of this $8 \%$ is as follows. About $7 \%$ are spiral galaxies that have naturally run out of gas without an obvious external trigger, and the other $1 \%$ are red because they are dusty. In conclusion, $3 \%$ of the red sequence, a rather low fraction, is red because of dust.

The shutdown of star formation when the halo mass grows above $M_{\text {crit }}$ introduces the notion of an 'entry mass' to the red sequence, $M_{\mathrm{star}}^{\mathrm{crit}}$, that is the typical stellar mass of the central galaxy of an $M_{\text {crit }}$ halo (Faber et al. 2007). The entry mass is predicted to be constant after $z \sim 2$ and higher at higher redshifts (Dekel \& Birnboim 2006). Its value at $z \lesssim 2$ is under $10 \%$ of $M_{\text {shock }} \sim 10^{12} M_{\odot}$, given the universal baryonic fraction of $\sim 0.15$ and assuming that at least half of the gas is prevented from making stars due to stellar feedback.

We illustrate the connection between shutdown, entry mass and downsizing by comparing the halo and stellar mass growth histories of galaxies with different final stellar masses (Fig. 4). We consider four mass bins along the red sequence in the colour-mass diagram (corresponding to the four red parallelograms in Fig. 3) and select six galaxies at random from each bin. The mass intervals corresponding to the four bins cover a factor of forty in stellar mass, from $\sim 0.2 M_{\text {star }}^{\text {crit }}$ to $\sim 8 M_{\text {star }}^{\text {crit }}$.

All galaxies display an initial phase, in which the stellar mass grows rapidly, followed by shutdown and a passive phase, in which the stellar mass remains essentially constant (Fig. 4). The transition between the two phases is accompanied by a very fast reddening of the $U-B$ galaxy colour. This reddening is recognizable as a vertical jump upward in a diagram of colour vs. stellar mass (Fig. 5 left column). It can also be seen in the galaxies' evolutionary colour-magnitude tracks (Fig. 5. right column), as it corresponds to the point where the galaxies start fading and reddening. In 23 out of
24 galaxies, the transition from blue to red coincides with the time when the halo mass crosses $M_{\text {crit }}$. A slight decrease of the stellar mass in the passive phase is due to the death of old stars not being replaced by new ones. Growth in this phase is by dry mergers only, seen as sudden upwards turns after $M_{\text {star }}(z)$ has flattened (Fig. 4), in contrast with the smoother growth of $M_{\text {star }}$ due to star formation.

The most massive galaxies have final stellar masses in the range $10^{11.7} M_{\odot} \lesssim M_{\text {star }} \lesssim 10^{11.9} M_{\odot}$. In these galaxies, $M_{\text {star }} \gg M_{\text {star }}^{\text {crit }}$ because their haloes started forming very early and crossed $M_{\text {crit }}$ when the entry mass was larger than $M_{\text {star }}^{\text {crit }} \sim 10^{11} M_{\odot}$. The upturn of $M_{\text {crit }}(z)$ at $z \geqslant 3$ causes most of these giant galaxies to become red-and-dead almost simultaneously at $z \simeq 3.5-4$. Their post-quenching luminosity evolution is reasonably well described by a simple relation of the form

$M_{B} \sim-21.5-z \pm 0.5$

(diagonal solid line and $\pm 0.5 \mathrm{mag}$ dotted lines in Fig. 4). The haloes of these galaxies correspond to the sites of galaxy clusters at low redshifts.

Frequent mergers inside massive haloes provide an additional mechanism for the growth of giant galaxies that can raise their mass up to $M_{\text {star }} \sim 10^{12} M_{\odot}$. Indeed, five out of our six massive galaxies grow by a factor of $\sim 2-3$ through dry mergers after entering the red sequence. This late merging agrees with semianalytic modelling calculations by De Lucia et al. (2006) and De Lucia \& Blaizot (2007). A note of caution regarding the comparison of these predictions to observations is that the light associated with the growing stellar mass at the centres of galaxy clusters may be spread out across a non-negligible fraction of the cluster volume. Combined with observational limitations, this may lead to an underestimate of the stellar mass in brightest cluster galaxies at the centers of clusters (Gonzalez et al. 2005; Lauer et al. 2007; Faber et al. 2007).

The upper-intermediate mass bin in Fig. $4\left(10^{11.2} M_{\odot}<\right.$ $\left.M_{\text {star }}<10^{11.3} M_{\odot}\right)$ is populated by central galaxies in which $M_{\text {halo }}$ has crossed $M_{\text {crit }}$ at $z \lesssim 3$, where $M_{\text {crit }}=M_{\text {shock }}$ (Eq. 1). All of these galaxies enter the red sequence with a same stellar mass of $M_{\text {star }}^{\text {crit }} \sim 10^{11} M_{\odot}$. In both the high and the upper-intermediate mass bin there is a strong correlation between the final stellar mass and the redshift at which the galaxies become red. Galaxies that enter the red sequence earlier end up being more massive at $z \sim 0$, even when the entry mass is similar, because they have more time to grow through dry mergers along the red sequence.

In contrast, the two lower mass bins $\left(10^{10.7} M_{\odot}<\right.$ $M_{\text {star }}<10^{10.8} M_{\odot}$ and $\left.10^{10.2} M_{\odot}<M_{\text {star }}<10^{10.3} M_{\odot}\right)$ are dominated by satellite galaxies, identified in Fig. 4 by an abrupt big rise in their halo mass growth as they fall into a massive host halo. As satellites, they have a lower merging rate, with the growth by dry mergers along the red sequence playing a role only in a couple of the low-intermediate mass galaxies, and in none of the low-mass galaxies. For most of the low-mass galaxies, the transition to the red sequence corresponds to a merger of their dark matter halo into a halo more massive than $M_{\text {crit }}$, causing them to shut down as satellites. As the timings of these events are not strongly correlated with the halo mass before merging, the low-mass galaxies display a wide spread in quenching times, which are not directly related to final mass. 


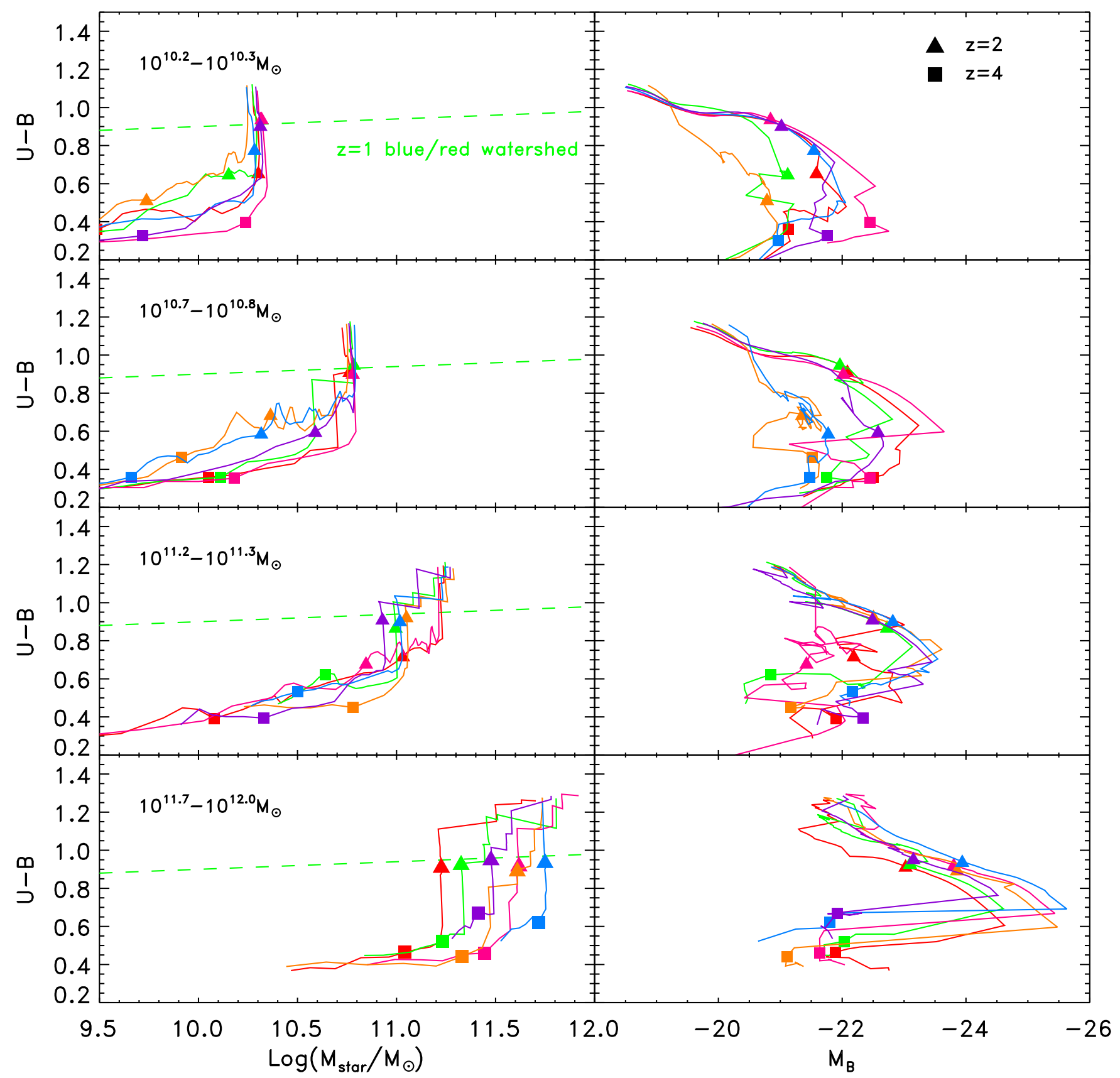

Figure 5. Time evolution in the colour-mass diagram for galaxies that are currently in four different mass bins along the red sequence, which are the same as in Fig. 3 and Fig. 4 The history tracks refer to the most massive progenitors. The colour assigned to each galaxy is the same as in Fig. 4 The green dashed line marks the green valley that separates the red sequence from the blue cloud at $z \sim 1$ in our model (see Fig. 6). The symbols refer to two different redshifts, as indicated, and show the pace at which galaxies move along the colour-mass tracks. Ignoring bursts of star formation, galaxies get steadily more massive and redder until they turn much redder in a short time once they are quenched. Small satellites are quenched and then keep a fixed mass. Massive galaxies continue to grow by dry mergers after they are quenched. The massive galaxies with $M_{\text {star }} \gtrsim 10^{11.7} M_{\odot}$ have already been quenched by $z=3$. Dry merging is not apparent at $M_{\text {star }} \lesssim 10^{11} M_{\odot}$. It becomes an important growth mechanism above this mass. We have plotted this figure assuming that all galactic discs are observed face-on. 
One lower-intermediate mass galaxy (light blue) resides in a halo less massive than $M_{\text {crit }}$ (and thus appears as one of the $7 \%$ blue symbols in Fig. 3). This galaxy has not been quenched by any of the three explicit mechanisms, but rather ran out of gas by continuous star formation.

One of the low-mass galaxies (fuchsia) has been the central galaxy of a halo that reached nearly $10^{13} M_{\odot}$ before it crossed $M_{\text {crit }}$ at $z \gtrsim 3$. However, this galaxy remained small because it was quenched before it had time to convert into stars the gas that had accreted and was accreting onto it.

To conclude, Fig. 4 demonstrates that shutdown at a constant critical halo mass introduces a rather constant entry mass for central galaxies into the red sequence. Central galaxies that enter the red sequence earlier end up more massive because they have more time for subsequent growth by dry merging. Note that the downsizing of $M_{\text {crit }}$ at high redshift predicted by Dekel \& Birnboim (2006) in itself induces a downsizing in the entry mass, which enhances the effect of archaeological downsizing in today's giant ellipticals. Dry merging is important in the growth of massive red galaxies with $M_{\text {star }} \geqslant 10^{11} M_{\odot}$. Galaxies that become satellites enter the red sequence with masses lower than the characteristic entry mass, and they dominate today's population at $M_{\text {star }} \ll 10^{11} M_{\odot}$.

\section{DOWNSIZING IN THE ASSEMBLY OF THE RED SEQUENCE}

In this section, we show that the shutdown above a critical halo mass produces downsizing in the buildup of the red sequence, in the sense that the high-mass end is populated at earlier times. For this purpose, we consider in Fig. 6] a large randomly selected sample of 8,000 galaxies, except that all the giant galaxies with $M_{\text {star }}>10^{11.5} M_{\odot}$ are included for better statistics. We plot a series of colour-mass diagrams, which show these galaxies at $z=0$, and their main progenitors at higher redshifts. The red symbols highlight the galaxies that end up on the red sequence in four distinct mass intervals, $\log M_{\text {star }}=[10,10.5],[10.5,11],[11,11.5],[11.5,12]$.

The overall population exhibits the same behaviour seen in Fig. 4 The distinction between a blue cloud and a red sequence is already present at $z \gtrsim 2$ (in agreement with FIRES observations; Giallongo et al. 2005), with about $60 \%$ of the most massive galaxies already on the red sequence at that redshift. Upper intermediate mass galaxies grow along the blue cloud until they reach the entry mass, near the upper mass limit for the blue cloud, where they turn red. The most massive galaxies climb up to the top of the blue sequence, turn red, then continue their growth by dry merging along the red sequence.

The mass growth due to dry mergers is a factor of $\sim 2.5$ in the highest mass bin and $\sim 1.7$ in the upper-intermediate mass bin. It becomes negligible below $10^{11} M_{\odot}$. This transition from wet to dry mergers may be related to the observed division of elliptical galaxies into two major types, giant boxy non-rotating ellipticals versus the smaller discy rotating ellipticals. Indeed, the origin of this distinction has been proposed to be the difference between dry merging along the red sequence and recent wet mergers along the blue cloud (Bender et al. 1988, 1989; Nieto \& Bender 1989; Nieto et al. 1991; Bender et al. 1992; Kormendy \& Bender
1996; Tremblay \& Merritt 1996; Gebhardt et al. 1996). The transition from wet to dry mergers may also be related to the division of ellipticals according to their inner density profiles, where giant ellipticals show cores and less massive ones show power-law cusps, possibly reflecting gas-rich mergers (Faber et al. 1997; Lauer et al. 2007). This transition is observed at $M_{V} \simeq-20.5$, which is $\sim 10^{10.8} M_{\odot}$ (assuming $M / L_{V} \simeq 6 \times 10^{-0.092\left(M_{V}+22\right)} M_{\odot} / L_{\odot}$ based on the $M / L$ estimates of Gebhardt et al. (2003)). This is indeed close to our estimated entry mass of $M_{\text {star }}^{\text {crit }} \sim 10^{11} M_{\odot}$, below which the growth is mainly by wet mergers.

The same transition mass is typically the minimum entry mass for central galaxies. It is also comparable to the bright edge of the blue cloud. The fact that this bright edge is constant with time reflects the fact that the entry mass for central galaxies is roughly constant after $z \sim 2$.

The low-mass bin and the lower intermediate mass bins, which are dominated by satellites, are mainly populated after $z \sim 1$. Low mass galaxies make an abrupt transition from blue to red at masses lower than the minimum entry mass for central galaxies. This is consistent with the notion that they are quenched when they fall into larger haloes and become members of groups or clusters.

We notice that at $z \sim 2$ the red sequence has a rather constant colour independent of mass, and that a tilt develops gradually in time. The origin of this effect, and its relation to downsizing, will be addressed elsewhere.

\section{ARCHAEOLOGICAL DOWNSIZING IN STELLAR AGES}

Perhaps the most useful way to measure downsizing is by comparing the distribution of stellar ages in galaxies of different masses. Thomas et al. (2005) deduced stellar ages from the absorption line indices $\mathrm{H} \beta, \mathrm{Mg} b$ and $<\mathrm{Fe}>$ for a sample of 124 early-type red sequence galaxies. They fitted the distribution of stellar ages in galaxies of a given stellar mass using a Gaussian, with the mean time and standard deviation as free parameters. Their main finding, as read from their Fig. 10, is that the mean stellar age is increasing with mass, while the scatter is decreasing with mass. They also find that the mean stellar age is increasing with environment density at a given stellar mass. It is important to realize that the measured age spreads are inferred from $[\alpha / \mathrm{Fe}]$ and not from detailed modelling of galaxy spectra. Moreover, the spread in the times at which different galaxies attain their peak SFR may be significantly larger than the age spread in each galaxy, which is what $[\alpha / \mathrm{Fe}]$ measures. Taking these differences into account is important when we compare the age spread estimated by Thomas et al. (2005) with those obtained from the mean star formation histories in our model.

To compare our model with the results by Thomas et al. (2005), we should consider all the stars that end up in a given red galaxy, and not just those that formed in its most massive progenitor. Fig. 7 plots the predictions of our model for the distribution of stellar ages in the spheroids of bulgedominated galaxies. We compare the results for the standard model (no shutdown, except in satellites) and the new model (shutdown of star formation in satellites, in massive haloes and in bulge-dominated galaxies). Once again, we split the 


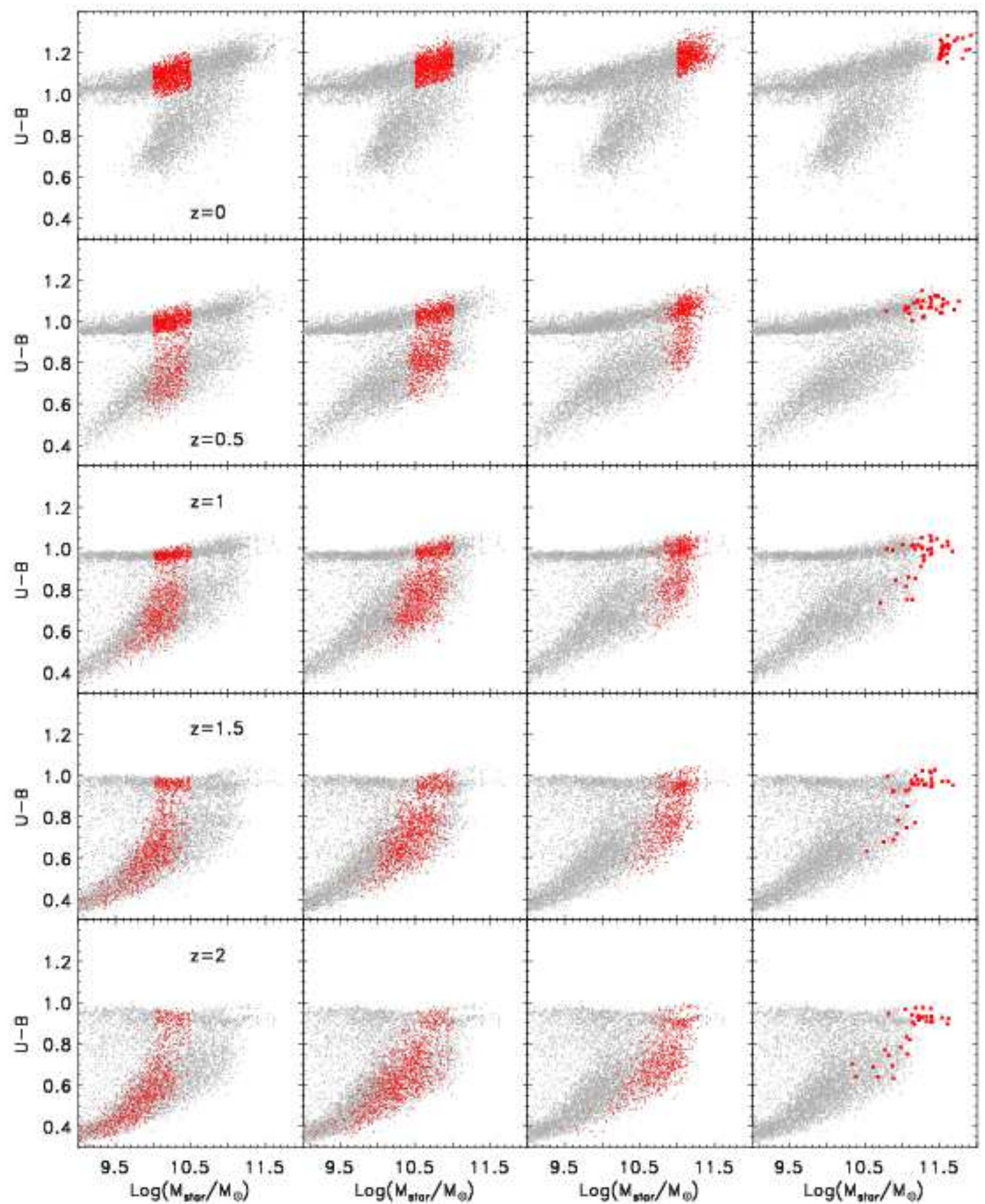

Figure 6. Evolution of the colour-mass diagram from $z \sim 2$ to $z \sim 0$. The grey dots represent 8,000 randomly selected galaxies at $z=0$ (and all the giant galaxies), and their main progenitors at higher redshifts. The galaxies with $M_{\text {star }}>10^{10} M_{\odot}$ on the red sequence at $z=0$ are divided into four bins according to their final stellar mass, as marked by the red symbols in the four columns of the upper row. The red symbols in the lower panels highlight the corresponding main progenitors at the different redshifts. 

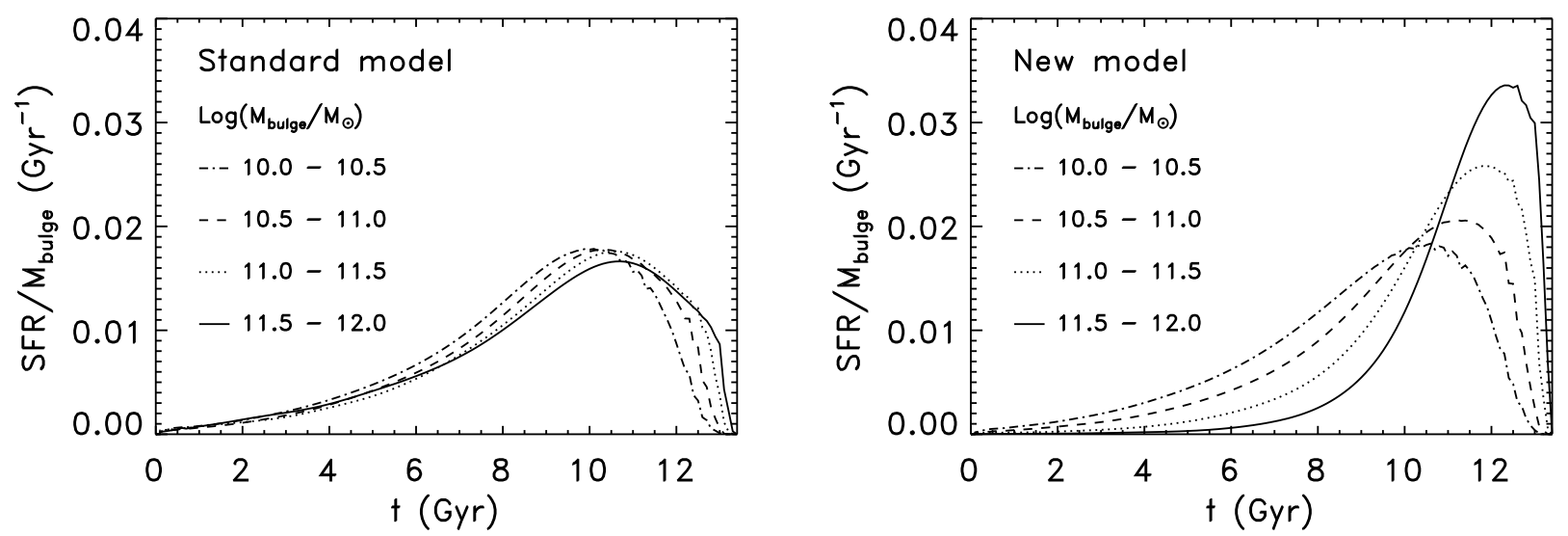

Figure 7. The distribution of stellar ages as a function of mass for bulge-dominated galaxies, obtained by averaging the specific SFR histories in the given mass bins. The specific SFR is inferred from the age distribution of all the stars in today's galaxies (not just those in their main progenitors). Left: the standard model. Right: the new model, in which star formation is shut down above a critical mass and in bulge-dominated central galaxies.

red galaxies into four stellar mass bins. We see that, in the standard model, galaxies of all masses have similar star formation histories. The weak downsizing signature probably reflects the natural downsizing of dark haloes in which star formation occurs only above a minimum halo mass (Neistein et al. 2006). In contrast, the new model shows a strong downsizing effect, where more massive galaxies form stars earlier and over a shorter time span, as observed.

In both models, the SFR histories for all masses are predicted to have long tails extending to the present time. This has also been seen in the semi-analytic simulations by De Lucia et al. (2006). Note, however, that the curves in Fig. 7 are averages over many galaxies. Individual galaxies have a narrower age spread. The general skewness of the SFR toward early times reflects the overall trend of the star formation history in the Universe, where roughly half the stars are made before a look-back time of $t=8 \mathrm{Gyr}(z=1)$. In the new model, the SFR in massive galaxies is truncated by crossing $M_{\text {crit }}$ early. Less massive central galaxies pass through $M_{\text {crit }}$ later, so their star formation stops later.

The two lowest mass bins have the widest age spreads because satellites fall into bigger haloes and get quenched over a wide range of times. Small differences between the two lowest mass bins reflect both the residual effect of central galaxies in the lower intermediate mass bin and the tendency of more massive satellites to fall into massive haloes earlier.

Fig. 8 compares the results of the new model with the mean stellar ages and internal age spreads inferred by Thomas et al. (2005), including the environment dependence. The model largely reproduces the observed effects. In the two environments, the model mean stellar ages increase with galaxy mass in general agreement with the observed trends, except that the model has a slight tendency to overestimate the stellar ages in the field (see below), but the big difference between environments may be an artifact of the small sample used (D. Thomas, private communication). In our model, the stellar populations of the most massive ellipticals are as old or even slightly older than those in Thomas et al. (2005), even in the densest environments. The model has no trouble making old-enough stars in massive

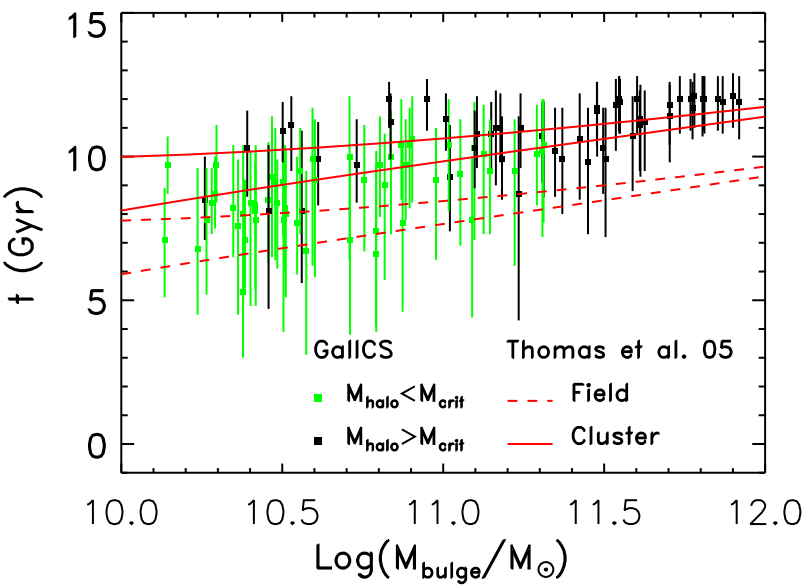

Figure 8. The median stellar age of bulges in bulge-dominated $(\mathrm{E} / \mathrm{S} 0)$ galaxies as a function of mass. The symbols and error bars correspond to the look-back time by which $(50 \pm 34) \%$ of the bulge stellar mass has formed. To mimic environment density, the green and black symbols refer to galaxies in haloes below and above the critical mass $\sim 2 \times 10^{12} M_{\odot}$, respectively. The red lines show in comparison the $\pm \sigma$ age spread as inferred by Thomas et al. (2005) for E/S0 galaxies in field and cluster environments.

galaxies. At any given galaxy mass, the age of the stellar population is higher in a cluster environment than in the field.

The internal spread in stellar ages is narrower for more massive galaxies, both in the model and the data. While in the model the most massive galaxies formed all their stars in a short time span $\lesssim 2$ Gyr, this time span is not as brief as that inferred by Thomas et al. (2005) from their measured values of $[\alpha / \mathrm{Fe}]$, which is $\sim 0.3 \mathrm{Gyr}$. At a time of $t \sim 2 \mathrm{Gyr}$ after the Big Bang, the characteristic dynamical time for flowing at the virial velocity from the virial radius to the halo centre is $\sim 0.4$ Gyr. The observed duration thus implies a massive burst of star formation on the shortest possible dynamical time while the model predicts a longer duration, 
which is comparable to the Hubble time at that epoch. A burst as short as inferred by Thomas et al. (2005) requires a process that accelerates the SFR and then rapidly shuts it down, as it could possibly be obtained by major mergers, quasar feedback, or shocked accretion Birnboim et al. 2007). Such a process is not incorporated in our current model.

The observed mean stellar ages are expected to be slightly lower than the model predictions for several reasons. First, the observed light-weighted average ages are younger than the model mass-weighted averages because the younger stars are brighter and dominate the light. Furthermore, younger populations have stronger Balmer equivalent widths, which dominate the total equivalent widths, and thus add to the brightness effect just mentioned, making light-weighted ages still younger. Finally, young tails in the age distribution, if present in real galaxies, would pull the peak of the Gaussian fit toward younger ages. Considering these limitations, the agreement between model and data is good.

\section{DOWNSIZING IN STAR FORMATION VS. UPSIZING IN ASSEMBLY}

In Fig. 9 we extend the analysis of the new model to other times that characterize the SFR and its shutdown. These are the times (a) of peak SFR, (b) of shutdown, (c) at which $M_{\text {halo }}$ crosses $M_{\text {crit }}$, (d) at which the galaxy becomes bulge dominated, and (e) at which the main progenitor attains half the final galaxy stellar mass. Each characteristic time can be interpreted as showing either downsizing or upsizing depending on whether it is monotonically decreasing or increasing as a function of final stellar mass. We shall see that all the above time indicators referring to star formation show a general downsizing trend, as opposed to the upsizing trend that the model predicts for the analogous time indicators that characterize mass assembly.

It is worth noting the differences between Fig. 9 and Fig. 8 First, the times $t$ in Fig. 9 are from the Big Bang, while in Fig. 8 they were lookback times, that is $t_{0}-t$, where $t_{0}=13.7 \mathrm{Gyr}$ is the present age of the Universe in the cosmology used for this study. Second, Fig. 8 is constructed from a small number of galaxies, where the error bars correspond to the internal stellar age spreads in the individual galaxies, while Fig. 9 shows the entire red galaxy population in the new model. Third, the galaxies in Fig. 8 are all red bulges of bulge-dominated galaxies, while Fig. 9 shows all the red galaxies, and the times contain the contribution of the disc component if present. Fourth, Fig. 8 refers to all the stars in today's bulges, while Fig. 9 refers only to the stars in the galaxies' main progenitor back in time (the purple symbols in Fig. 9 are an exception).

The time of maximum SFR, $t_{\max \text { sfr }}$, is shown in Fig. 97. In most red galaxies, it occurred within the first $\sim 4 \mathrm{Gyr}$ from the Big Bang. There is a clear but weak downsizing trend in the epoch of maximum SFR. In the most massive galaxies, the SFR peaked at $t \sim 1-2 \mathrm{Gyr}$, while at low masses there is a larger spread in $t_{\text {max sfr. We thus recover }}$ downsizing features similar to the 'archaeological' downsizing obtained from the stellar age distribution in red galaxies at $z \sim 0$.

(C) 2005 RAS, MNRAS 000, 120

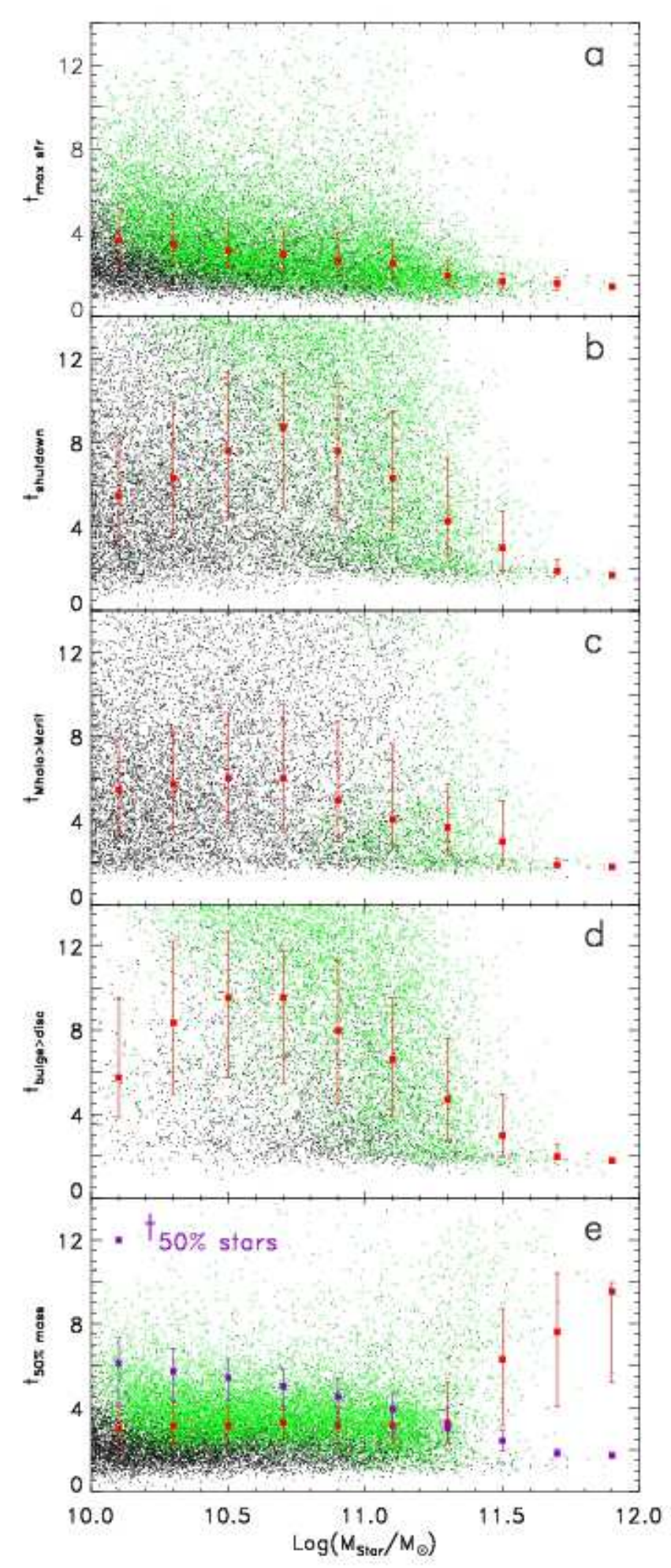

Figure 9. Times characterizing the SFR and its shutdown versus final stellar mass. (a) The time of peak SFR $t_{\max s f r}$. (b) The time of star formation shutdown $t_{\text {shutdown }}$ (c) The time at which $M_{\text {halo }}$ crosses $M_{\text {crit }}, t_{\text {Mhalo }}>$ Mcrit. (d) The time at which the bulge mass becomes larger than the disc mass $t_{\mathrm{bulge}}>$ disc . (e) The assembly time at which the main progenitor attains $50 \%$ of the final stellar mass, $t_{50 \%}$ (red symbols). Also shown is the time at which half of the stars have formed (purple symbols). The times, measured from the Big Bang, are evaluated by tracking the main progenitor of each galaxy back in time. Central galaxies today are marked green and satellites are marked black. Red symbols with 


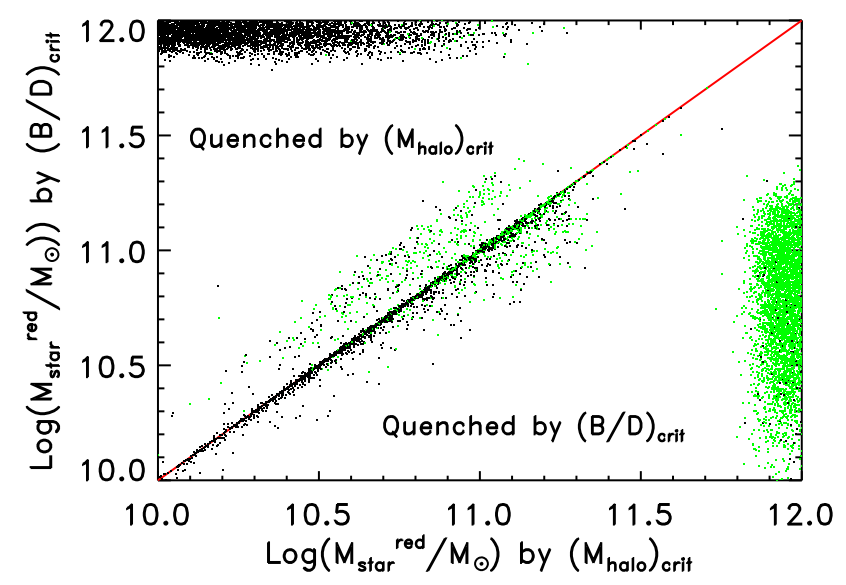

Figure 10. Role of the two quenching criteria. Each point shows the stellar masses of a galaxy when it became bulge dominated ( $y$-axis) and when its halo crossed $M_{\text {crit }}$ ( $x$-axis). Galaxies that crossed $M_{\text {crit }}$ before (after) they became bulge-dominated lie above (below) the diagonal red line. Green and black points refer to central and satellite galaxies at $z=0$, respectively. Galaxies that never become bulge-dominated, mainly satellites, are put near the top edge of the plot. Galaxies that never cross the critical halo mass, mainly centrals, are put near the right edge of the plot.

For a fixed final stellar mass, the SFR peak occurs earlier in satellite galaxies than in central galaxies. This is because low-mass central galaxies are in low mass haloes in which star formation does not start early, it is slowed down by supernova feedback, and it is not shut down early. Satellites, on the other hand, much like high stellar mass galaxies, can be part of massive haloes already at early times, so they can form stars early and also shut down early (SDSS data, Blanton et al. 2006; our new model, Cattaneo et al. 2006).

The time of shutdown in the most massive progenitor, $t_{\text {shutdown }}$, is shown in Fig. 9p. In $\sim 7 \%$ of the red galaxies with $M_{\text {star }}>10^{10} M_{\odot}$ the formation of stars has never been quenched by any of the explicit criteria applied in the new model. These galaxies have turned red simply because they have exhausted their gas reservoir. In addition, $\sim 2 \%$ of the red sequence at $M_{\text {star }}>10^{10} M_{\odot}$ is made of starbursts, which are red because they are dusty. We have removed these unquenched and dusty galaxies from Fig. 9b and from the shown median. The general downsizing trend in Fig. $9 \mathrm{~b}$ above $\sim 10^{10.7} M_{\odot}$ is similar to Fig. 97, especially for the central galaxies, but the magnitude of the effect is stronger

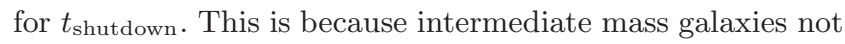
only reach their peak SFR somewhat later than more massive galaxies, but they also have tails of SFR extending to late times, $z \lesssim 1$ (Fig. 7). The most massive galaxies undergo an intense burst of star formation when the Universe is only $\sim 1.5$ Gyr old and then they are almost immediately shut down. In red galaxies of intermediate masses, the peak SFR is at $t \sim 3 \mathrm{Gyr}$ and the formation of stars drags on for $\sim 6$ Gyr after the peak. The shutdown time does not show downsizing at low masses, where the red population is dominated by satellites.

We recall that our model contains two quenching mechanisms. One is due to the shock heating in haloes once they

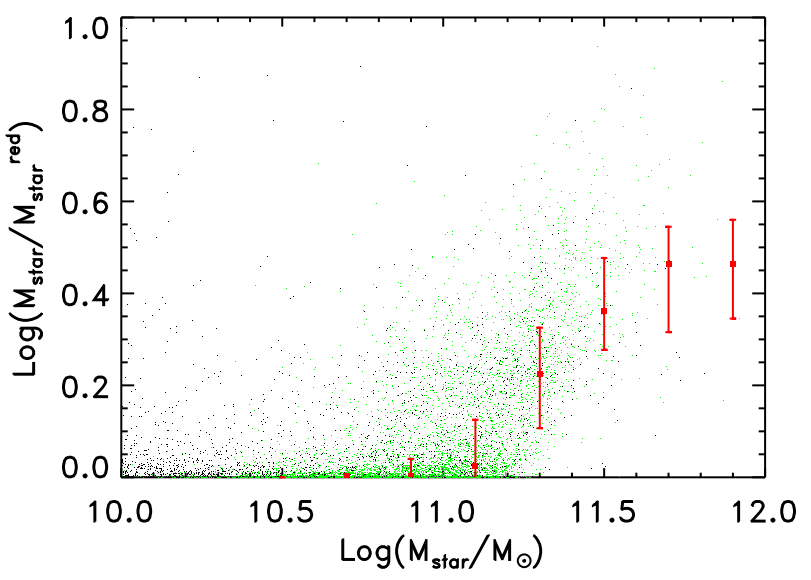

Figure 11. Growth by dry merging along the red sequence versus stellar mass. It is measured by the ratio between the final stellar mass of a red galaxy and the mass it had when it was quenched by one of the three shutdown mechanisms of our model. Green and black points refer to central and satellite galaxies at $z=0$, respectively. The red symbols with error bars show the median values of $M_{\text {star }} / M_{\text {star }}^{\text {red }}$ and the lower and upper quartiles in logarithmic bin of $0.2 \mathrm{dex}$ in $M_{\text {star }}$.

become more massive than $M_{\text {crit }}$, and the other is a result of wet major mergers. Mergers are assumed to simultaneously produce dominant stellar spheroids and trigger quasars at their centres, which then help removing the gas available for star formation in merger remnants (e.g., Springel et al. 2005a). The bulge-dominance criterion applied in the the new model is meant to mimic quasar feedback of this sort. We assess the relative importance of these two mechanisms in the new model by splitting Fig. 9b into two, showing separately the times for crossing the critical halo mass

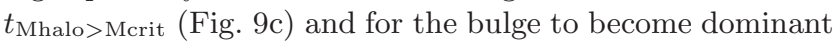
$t_{\text {bulge }>\text { disc }}$ (Fig. 9d). Galaxies that have never crossed the critical halo mass do not appear in Fig. 95, and galaxies that have never become bulge-dominated do not appear in Fig. 9 d.

Massive galaxies with $M_{\text {star }}>10^{11} M_{\odot}$ and $t_{\text {shutdown }}<$ 3 Gyr in Fig. 9b populate the same region of the diagram in Fig. 9r and Fig. 9d. These are central galaxies of massive haloes, which simultaneously obey $M_{\text {halo }}>M_{\text {crit }}$ and have a dominant spheroid due to intense merging. Galaxies that are less massive tend to show up either in Fig. 96 or in Fig. 9d, depending on whether they are satellites or small central red galaxies.

The same three populations can be identified in Fig. 10. which displays for every galaxy the stellar masses at the two characteristic times: when it entered a halo more massive than the critical mass, and when it became bulge-dominated. The cloud of black dots at the very top consists of satellite galaxies in massive haloes, which have never become bulge-dominated because the merger rate is low for satellites. This population corresponds to the black symbols in Fig. 9b that survive in Fig. 9r but not in Fig. 9d. The cloud of green dots at the very right consists of bulge-dominated central galaxies in haloes that are just below the critical mass. These galaxies correspond to the intermediate mass galaxies shown as green symbols in Fig. 3. Without the 
bulge shutdown criterion, these galaxies would be on the blue cloud, and they would extend it to higher luminosities (Cattaneo et al. 2006). This population corresponds to the green symbols in Fig. 9b that survive in Fig. 9d but not in Fig. 9. The third population comprises galaxies that both inhabit massive haloes and are bulge-dominated. This population contains the most massive objects and is composed of both central and satellite galaxies. The distinction between central and satellite galaxies is not very useful here because many satellites were central until recently. A more useful distinction is between the galaxies that were first quenched by the bulge criterion and the galaxies that were first quenched by the halo criterion (points below and above the diagonal respectively). These galaxies obey the two quenching criteria almost back-to-back because the two events are related to the hierarchical growth of dark matter haloes.

The characteristic times considered so far are indicators of star-formation history. Fig. 9e displays instead an indicator of the assembly history, the time $t_{50 \% \text { mass }}$ at which $50 \%$ of the final stellar mass has been assembled in a single objects (red symbols). The assembly time is roughly constant with mass for low and intermediate-mass galaxies, and it actually reveals upsizing at the high-mass end. This resembles the assembly upsizing of the main progenitor of dark matter haloes in Neistein et al. (2006).

In galaxies of low or intermediate mass, $t_{50 \% \text { mass }}$ is comparable to $t_{\max \text { sfr }}$ of Fig. 9a. The complete shutdown of star formation occurs when the Universe is $\gtrsim 8 \mathrm{Gyr}$ old, but $\gtrsim 80 \%$ of the final stellar mass is already in place in the most massive progenitor after the first $\sim 5$ Gyr. Only little mass is added to galaxies of $M_{\text {star }}<10^{11} M_{\odot}$ after this point, as the merger rate is not high in this mass range, as demonstrated by Fig. 11 which shows the mass growth factor after entering the red sequence as a function of the final stellar mass. Moreover, accretion onto the haloes of small galaxies, and therefore onto small galaxies themselves, is suppressed by tidal effects in the vicinity of more massive haloes (Hahn, Dekel et al., in preparation).

In contrast to small galaxies, galaxies with $M_{\text {star }} \gtrsim 10^{11} M_{\odot}$ experience substantial growth through dry mergers after they cease to form stars (Fig. 11). Therefore, the most massive galaxies continue gathering mass for a much longer time. When the Universe is $\sim 7$ Gyr old, they have barely put together half of their final stellar mass.

The onset of upsizing in assembly is right above the minimum entry mass for central galaxies, near the mass separating the boxy and discy ellipticals (Section 4). Our model hints to a simple explanation for this bimodality of structural and kinematic properties of galaxies along the red sequence. Discy ellipticals are the products of recent wet mergers of gaseous disks along the blue cloud, in haloes of $M_{\text {halo }}<M_{\text {crit }}$. Boxy ellipticals, on the other hand, result from dry mergers along the red sequence, typically at the centres of haloes with $M_{\text {halo }}>M_{\text {crit }}$. The most massive ellipticals, which have only finished assembling $80 \%$ of their final stellar mass in the last few billion years, are to be identified with the brightest cluster galaxies at the centres of Abell clusters. Also see de Lucia \& Blaizot (2007) for a semi-analytic study.

Fig. 9. also displays for comparison the time at which $50 \%$ of the final stellar mass was formed (purple symbols), showing a star-formation downsizing similar to the trends

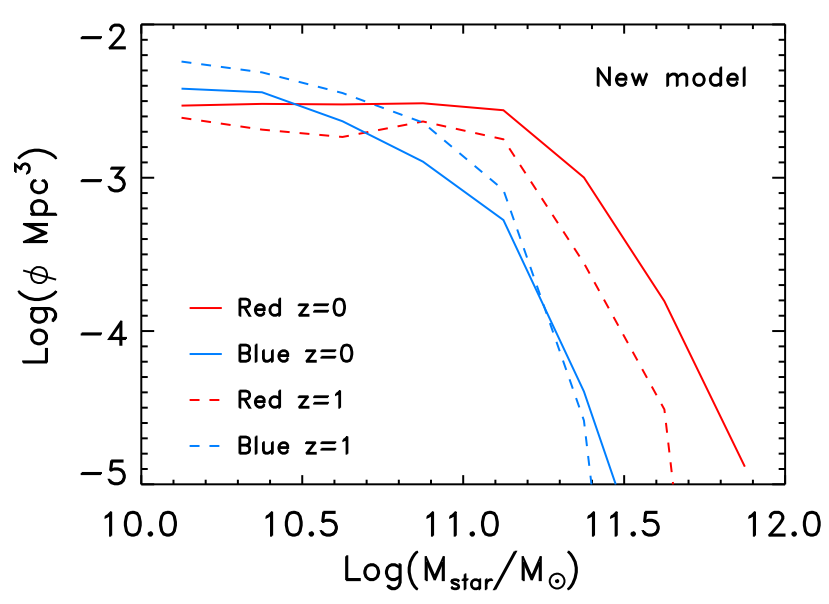

Figure 12. The evolution of the mass functions of red and blue galaxies from $z=1$ (dashed lines) to $z=0$ (solid lines) in our model with star formation shutdown.

seen in the other panels. This figure demonstrates how upsizing in assembly coexists with downsizing in star formation in the same hierarchical clustering scenario.

A second final remark on Fig. 9 concerns the difference between central and satellite galaxies. Compared to satellites of the same mass, in central galaxies the SFR peaks later, it shuts down later, and the stellar mass assembles later (compare the green and black dots in Fig. 97, b and e).

\section{DOWNSIZING IN THE GALAXY MASS FUNCTIONS?}

The time evolution of the mass functions of red and blue galaxies has been used as another indicator for downsizing in the formation of the red sequence. The features considered, for example, are (a) the downward shift with time of the mass where the red and blue functions cross each other, and (b) the indications for a late increase in the mass function of red galaxies at low masses compared to an early increase at high masses Bundy et al. 2005; Borch et al. 2006; Pannella et al. 2006; also see the discussion in Faber et al. 2007). In this section we highlight uncertainties in such arguments, both due to inherent difficulties in interpreting model mass functions and to the large uncertainties that still exist in the data themselves. Our principal conclusion is that the mass functions of red and blue galaxies are poor indicators of downsizing.

The observational uncertainties are discussed separately in the Appendix. Here we concentrate on the predictions of the model and demonstrate how hard it is to use mass functions to infer downsizing or upsizing, even if the data were perfectly accurate.

The evolution of the mass functions of red and blue galaxies in the new model from $z=1$ to $z=0$ is shown in Fig. 12. The red mass function has grown at all masses, while the blue function has decreased or remained constant. Below $M_{\text {star }}^{\text {crit }}$ there is a rather uniform growth of the red function by $\sim 0.2$ dex at all masses, accompanied by a comparable uniform decrease in the blue function over the same mass 
range. Above $M_{\text {star }}^{\text {crit }}$, the red function grows by a larger factor while the blue function remains roughly constant.

Our earlier analysis can help us identify the processes responsible for the buildup of the red sequence at the lowand high-mass ends. Red galaxies with $M_{\text {star }}>M_{\text {star }}^{\text {crit }}$ consist of stars that have formed at $z \gg 1$, so their progenitors were already on the red sequence at $z \sim 1$. These galaxies have continued assembling through dry mergers of red galaxies, such that the growth of the red population at the high-mass end comes largely at the expense of the red population at the low-mass end, from below $M_{\text {star }}^{\text {crit }}$. The true influx of galaxies into the low-mass red population equals the increase of the red mass function seen in Fig. 12 plus the transfer of red galaxies to the high-mass end by dry mergers. This influx must come from blue galaxies migrating to the red sequence because the increase in total stellar mass in red galaxies cannot come from new star formation in the red sequence (Faber et al. 2007).

Part of the stellar mass transferred from the blue to the red sequence is readily accounted for by the decrease in the number of blue galaxies at $M_{\text {star }}<M_{\text {star }}^{\text {crit }}$, where most of the blue sequence mass resides. However, the total stellar mass in galaxies with $M_{\text {star }}>10^{10} M_{\odot}$ has increased from $z \sim 1$ to $z \sim 0$. This implies that, despite the drop in the number of blue galaxies, new stellar mass must have been formed along the blue sequence at $0 \lesssim z \lesssim 1$. In our model, this is mainly due to the conversion of disc gas into stars. Only to a lesser extent is it due to new galaxies moving into the blue sequence due to growth through the accretion of gas and wet mergers.

We have thus identified three fluxes that drive the evolution of the populations of red and blue galaxies in the colour-mass diagram from $z \sim 1$ to $z \sim 0$. First, the flux of stellar mass into the blue sequence due to conversion of gas into stars in blue galaxies $\lesssim M_{\text {star }}^{\text {crit }}$. Second, the transition of blue galaxies onto the red sequence at $\lesssim M_{\text {star }}^{\text {crit }}$. Third, the transfer of red galaxies by dry mergers from the low-mass part of the red sequence to the high-mass part, $M_{\text {star }}>M_{\text {star }}^{\text {crit }}$. Only the first mode generates new stellar mass in the overall galaxy population, and only the first two contribute to the growth of stellar mass in the red sequence.

Now, with the benefit of hindsight, we are ready to interpret the changes seen in Fig. 12 . One obvious trend in both the model and the figure is the assembly upsizing that creates the most massive elliptical galaxies on the red sequence at late times. This is visible in figure as the rightward shift in the red function at high masses. These galaxies have assembled late and therefore appear at high masses late. Less clear, however, is the behaviour of the red mass function below $M_{\text {star }}^{\text {crit }}$. We know from the detailed histories of galaxies in Fig. 9 that the star-formation downsizing trend at these masses is strong, yet this effect is not obvious in the mass functions. All masses below $M_{\text {star }}^{\text {crit }}$ appear to grow about the same. To be precise, there does not appear to be much greater growth at the lowest masses on the red sequence compared to the growth at $M_{\text {star }}^{\text {crit }}$. This happens largely because our model contain many satellite disc galaxies that form too early and are quenched too early (Fig. A1 in the Appendix). The lesson is that deducing either downor upsizing trends from the mass function is tricky.

Fig. 12 also sheds light on a common misinterpretation of the crossover mass, that is, the point where the red function intersects the blue function. Downward movement of this point in time has been suggested as an indicator of downsizing (Bundy et al. 2006; Hopkins et al. 2007). Even though downsizing is actually taking place at these masses in the model, we have seen that this is not evident from each of the mass functions alone. Instead, Fig. 12 shows quite clearly that the crossover point is moving downward simply because the number of red galaxies is growing relative to the number of blue galaxies, independent of how these changes depend on mass. This effect is enhanced when the mass functions are relatively flat, which is true both in the model and in real data (see the Appendix). The crossover point would move downward even when blue galaxies become red in a uniform rate at all masses, namely without any real downsizing trend in the SFR along the blue cloud or in the buildup of the red sequence.

In the Appendix, we make three points concerning the observed mass functions. First, determining the number of very massive galaxies at the steep bright end is tricky because even small random mass errors create large errors in the counts. The observed mass function at the bright end is not the true function but rather the true function smoothed by errors. Owing to the steepness of the bright end, this tends to falsely inflate the number of massive objects (the socalled Eddington effect; see also Kitzbichler \& White 2006 and Lauer et al. 2007). The Appendix shows that random rms errors of only 0.2 dex in mass inflate the observed counts by a full order of magnitude at $M_{\text {star }} \sim 10^{11.5} M_{\odot}$. Model counts must therefore be smoothed to mimic this effect before comparing to data, but it is unclear whether the errors get smaller or larger with redshift, and thus whether the evolutionary effect is positive or negative.

Second, the total magnitudes of massive ellipticals may be systematically underestimated because the light profiles of very massive galaxies are nearly isothermal and it is hard to estimate the total light, let alone distinguish galaxy light from intracluster light. This problem likely worsens at low redshifts, and so the sense is probably to underestimate galaxy masses, and thus the number of massive galaxies towards low redshifts.

Third, before comparing mass functions from different authors, it is necessary to convert them to the same assumed stellar initial mass function (IMF). Certain authors who formerly appeared to agree now disagree after this correction is made. Consistent trends in even just the raw counts are not yet evident.

Given these difficulties, both observational and theoretical, we do not attempt in this paper to deduce either down- or upsizing trends from luminosity functions or mass functions. All that can be said is that the number of red galaxies has increased by at least a factor of 2 near $L_{*}$ and $M_{*}$ since $z=1$, while the number of blue galaxies has not increased since $z=1$ (Faber et al. 2007). These trends do not alone argue for down- or upsizing, but at least they are in reasonable agreement with the results of our model.

\section{DISCUSSION AND CONCLUSION}

We showed that the downsizing of elliptical galaxies, where the more massive galaxies formed their stars earlier and over a shorter period, is not in conflict with the standard hi- 
erarchical clustering scenario. We demonstrated that this is in fact a natural outcome of the shutdown of star formation in haloes above a critical mass $M_{\text {crit }} \sim 10^{12} M_{\odot}$. Such a shutdown is essential for explaining the distribution of galaxies in luminosity and colour (Cattaneo et al. 2006; Bower et al. 2006; Croton et al. 2006). In particular, this shutdown prevents the overgrowth of the central galaxies in massive haloes beyond the observed upper limit for bright blue galaxies, and allows the appearance of massive red and dead galaxies, thus reproducing the observed galaxy bimodality. The critical mass for shutdown originates from stable virial shock heating, which occurs in haloes above this threshold and shuts down the cold gas supply for star formation there (Birnboim \& Dekel 2003; Binnev 2004; Kereš et al. 2005; Dekel \& Birnboim 2006). This scenario only works if gas in massive haloes is maintained hot by a continuous energy source that balances the radiative cooling over long cosmological periods. The mechanical energy of jets from low-power radio AGNs is a promising candidate (Fabian et al. 2003; Ruszkowski et al. 2004; Forman et al. 2005; Best et al.| 2005; Voit \& Donahue 2005; Best et al. 2006; Dunn \& Fabian 2006), which can result in self-regulated accretion and AGN activity capable of maintaining the required long-term shutdown (Omma \& Binnev 2004; Rafferty et al. 2006; Cattaneo \& Teyssier 2007). An alternative/additional source of long-term quenching is the gravitational energy of accretion into the centres of the potential wells of dark matter haloes (Birnboim et al. 2007; Dekel \& Birnboim 2007; Khochfar \& Ostriker 2007).

The shutdown at a critical halo mass introduces an entry mass $M_{\text {star }}^{\text {crit }}$ to the red sequence. Central galaxies cease to make stars and turn red and dead at a mass equal to the characteristic mass of the central galaxy in a halo of mass $M_{\text {crit }}$. The central galaxies of the most massive haloes reach $M_{\text {star }}^{\text {crit }} \sim 10^{11} M_{\odot}$ earlier (Fig. 4). Therefore, they have more time to grow above $M_{\text {star }}^{\text {crit }}$ by dry merging along the red sequence. For this reason, the central galaxies of the most massive haloes are the most massive galaxies in the Universe and those that contain the oldest stellar populations. In our model, the early growth of the central galaxies of the most massive haloes is also helped by the fact that $M_{\text {crit }}$ is assumed to be higher than $2 \times 10^{12} M_{\odot}$ before $z \gtrsim 3$, based on the predictions of Dekel \& Birnboim (2006).

We demonstrated that shutdown-driven downsizing is in agreement with the observed archaeological downsizing of elliptical galaxies, as measured by Thomas et al. (2005; Fig. 8). The introduction of shutdown above a threshold mass also explains why the emergence of the red sequence has started already at $z \sim 2$ and why the shutdown begins at high masses (Fig. 6). Over 3/4 of the model ellipticals with $M_{\text {star }}>3 \times 10^{11} M_{\odot}$ were already on the red sequence by $z \sim 2$, in agreement with FIRES observations (Giallongo et al. 2005). In contrast, the buildup of the red sequence at $M_{\text {star }} \lesssim 10^{11} M_{\odot}$ continues at $z \lesssim 1$.

The downsizing in star formation and the upsizing in mass assembly affect the evolution of the mass functions of red and blue galaxies. The three phenomena that control the evolution of the mass functions are: the formation of new stellar mass in blue sequence galaxies, the migration of blue galaxies onto the red sequence, and the migration of red galaxies from the low-mass end to the high-mass end of the red sequence due to dry merging. However, the combi- nation of downsizing in star formation and upsizing in mass assembly is such that the evolution of the mass functions is a poor indicator of downsizing even if the data were perfectly accurate (the large uncertainties that still exist in the data themselves have been discussed separately in Appendix A).

Giant ellipticals grow by $\sim 0.4-0.5$ dex after entering the red sequence and by $\sim 0.2-0.3$ dex between $z \sim 1$ and $z \sim 0$. Within the red population, they are those that have finished to assemble their mass most recently (Fig. 98, red symbols; also De Lucia et al. 2006 and De Lucia \& Blaizot 2007), despite the fact that their final mass was turned into stars earlier than in other galaxies (Fig. 9e, purple symbols), due to the early shutdown of star formation in these objects.

In this paper we have focussed on the contribution of the shutdown above a critical halo mass to the downsizing. In principle,there could be other reasons for the observed downsizing. Nevertheless, the comparison of the stellar ages in the two models, which differ only by imposing an explicit shutdown, provides clean evidence that a shutdown of this sort can be the main driver of the observed downsizing.

\section{ACKNOWLEDGMENTS}

We acknowledge stimulating discussions with J. Devriendt, M. Pannella and R. Somerville. The simulations presented in this article were run on the Horizon supercomputer at the CRAL in Lyon within the framework of the Horizon Project. This research has been partly supported by ISF 213/02, by GIF I-895-207.7/2005, by a France-Israel grant, by the Einstein Center at HU, and by NASA ATP NAG58218 .

\section{REFERENCES}

Bahcall N. A., 1977, ApJL, 218, L93

Baldry I. K., Glazebrook K., Brinkmann J., Ivezić Ž., Lupton R. H., Nichol R. C., Szalay A. S., 2004, ApJ, 600, 681

Balogh M. L., Baldry I. K., Nichol R., Miller C., Bower R., Glazebrook K., 2004, ApJL, 615, L101

Barger A. J., Cowie L. L., Mushotzky R. F., Yang Y., Wang W.-H., Steffen A. T., Capak P., 2005, AJ, 129, 578

Bell E. F., de Jong R. S., 2001, ApJ, 550, 212

Bell E. F., McIntosh D. H., Katz N., Weinberg M. D., 2003, ApJS, 149, 289

Bell E. F., Papovich C., Wolf C., Le Floc'h E., Caldwell J. A. R., Barden M., Egami E., McIntosh D. H., Meisenheimer K., Pérez-González P. G., Rieke G. H., Rieke M. J., Rigby J. R., Rix H.-W., 2005, ApJ, 625, 23

Bell E. F., Wolf C., Meisenheimer K., Rix H., Borch A., Dye S., Kleinheinrich M., Wisotzki L., McIntosh D. H., 2004, ApJ, 608, 752

Bender R., Burstein D., Faber S. M., 1992, ApJ, 399, 462

Bender R., Doebereiner S., Moellenhoff C., 1988, A\&AS, 74, 385

Bender R., Surma P., Doebereiner S., Moellenhoff C., Madejsky R., 1989, A\&A, 217, 35

Best P. N., Kaiser C. R., Heckman T. M., Kauffmann G., 2006, MNRAS, 368, L67 
Best P. N., Kauffmann G., Heckman T. M., Brinchmann J., Charlot S., Ivezić Ž., White S. D. M., 2005, MNRAS, 362,25

Binney J., 1977, ApJ, 215, 483

Binney J., 2004, MNRAS, 347, 1093

Birnboim Y., Dekel A., 2003, MNRAS, 345, 349

Birnboim Y., Dekel A., Neistein E., 2007, MNRAS, 380, 339

Blanton M. R., Eisenstein D. J., Hogg D. W., Zehavi I., 2006, astro-ph/0411037

Blumenthal G. R., Faber S. M., Primack J. R., Rees M. J., 1984, Nature, 311, 517

Borch A., Meisenheimer K., Bell E. F., Rix H.-W., Wolf C., Dye S., Kleinheinrich M., Kovacs Z., Wisotzki L., 2006, A\&A, 453, 869

Bower R. G., Benson A. J., Malbon R., Helly J. C., Frenk C. S., Baugh C. M., Cole S., Lacey C. G., 2006, pre-print astro-ph/0511338

Brinchmann J., Ellis R. S., 2000, ApJL, 536, L77

Bundy K., Ellis R. S., Conselice C. J., 2005, ApJ, 625, 621 Bundy K., Ellis R. S., Conselice C. J., Taylor J. E., Cooper M. C., Willmer C. N. A., Weiner B. J., Coil A. L., Noeske

K. G., Eisenhardt P. R. M., 2006, ApJ, 651, 120

Cattaneo A., Blaizot J., Devriendt J., Guiderdoni B., 2005, MNRAS, 364, 407

Cattaneo A., Blaizot J., Weinberg D. H., Kereš D., Colombi S., Davé R., Devriendt J., Guiderdoni B., Katz N., 2007, MNRAS, 377, 63

Cattaneo A., Combes F., Colombi S., Bertin E., Melchior A.-L., 2005, MNRAS, 359, 1237

Cattaneo A., Dekel A., Devriendt J., Guiderdoni B., Blaizot J., 2006, MNRAS, pp 780-+

Cattaneo A., Haehnelt M. G., Rees M. J., 1999, MNRAS, 308, 77

Cattaneo A., Teyssier R., 2007, MNRAS, 376, 1547

Cole S., Lacey C. G., Baugh C. M., Frenk C. S., 2000, MNRAS, 319, 168

Cowie L. L., Songaila A., Hu E. M., Cohen J. G., 1996, AJ, 112,839

Cox T. J., Dutta S. N., Di Matteo T., Hernquist L., Hopkins P. F., Robertson B., Springel V., 2006, ApJ, 650, 791

Croton D. J., Springel V., White S. D. M., De Lucia G., Frenk C. S., Gao L., Jenkins A., Kauffmann G., Navarro J. F., Yoshida N., 2006, MNRAS, 365, 11

Davis M., Efstathiou G., Frenk C. S., White S. D. M., 1985, ApJ, 292, 371

De Lucia G., Blaizot J., 2007, MNRAS, 375, 2

de Lucia G., Blaizot J., 2007, MNRAS, 375, 2

De Lucia G., Springel V., White S. D. M., Croton D., Kauffmann G., 2006, MNRAS, 366, 499

Dekel A., Birnboim Y., 2006, pre-print astro-ph/0412300

Dekel A., Birnboim Y., 2007, ArXiv e-prints, 707

Dekel A., Silk J., 1986, ApJ, 303, 39

Devriendt J. E. G., Guiderdoni B., Sadat R., 1999, A\&A, 350,381

Drory N., Bender R., Feulner G., Gabasch A., Hopp U., Noll S., Pannella M., Saglia R. P., Salvato M., 2006, The Messenger, 125, 15

Drory N., Bender R., Feulner G., Hopp U., Maraston C., Snigula J., Hill G. J., 2004, ApJ, 608, 742

Drory N., Salvato M., Gabasch A., Bender R., Hopp U., Feulner G., Pannella M., 2005, ApJL, 619, L131
Dunn R. J. H., Fabian A. C., 2006, MNRAS, 373, 959

Faber S. M., Tremaine S., Ajhar E. A., Byun Y.-I., Dressler A., Gebhardt K., Grillmair C., Kormendy J., Lauer T. R., Richstone D., 1997, AJ, 114, 1771

Faber S. M., Willmer C. N. A., Wolf C., Koo D. C., Weiner B. J., Newman J. A., Im M., Coil A. L., Conroy C., Cooper M. C., Davis M., Finkbeiner D. P., Gerke B. F., Gebhardt K., Groth E. J., 2007, ApJ, 665, 265

Fabian A. C., Sanders J. S., Allen S. W., Crawford C. S., Iwasawa K., Johnstone R. M., Schmidt R. W., Taylor G. B., 2003, MNRAS, 344, L43

Forman W., Nulsen P., Heinz S., Owen F., Eilek J., Vikhlinin A., Markevitch M., Kraft R., Churazov E., Jones C., 2005, ApJ, 635, 894

Gallagher J. S., 1978, ApJ, 223, 386

Gebhardt K., Richstone D., Ajhar E. A., Lauer T. R., Byun Y.-I., Kormendy J., Dressler A., Faber S. M., Grillmair C., Tremaine S., 1996, AJ, 112, 105

Gebhardt K., Richstone D., Tremaine S., Lauer T. R., Bender R., Bower G., Dressler A., Faber S. M., Filippenko A. V., Green R., Grillmair C., Ho L. C., Kormendy J., Magorrian J., Pinkney J., 2003, ApJ, 583, 92

Giallongo E., Salimbeni S., Menci N., Zamorani G., Fontana A., Dickinson M., Cristiani S., Pozzetti L., 2005, ApJ, 622, 116

Gonzalez A. H., Zabludoff A. I., Zaritsky D., 2005, ApJ, 618, 195

Graham A. W., Erwin P., Caon N., Trujillo I., 2001, ApJL, 563, L11

Graves G. J., Faber S. M., Schiavon R. P., Yan R., 2007, ArXiv e-prints, 707

Guiderdoni B., Hivon E., Bouchet F. R., Maffei B., 1998, MNRAS, 295, 877

Guzman R., Gallego J., Koo D. C., Phillips A. C., Lowenthal J. D., Faber S. M., Illingworth G. D., Vogt N. P., 1997, ApJ, 489, 559

Hasinger G., Miyaji T., Schmidt M., 2005, A\&A, 441, 417

Hatton S., Devriendt J. E. G., Ninin S., Bouchet F. R., Guiderdoni B., Vibert D., 2003, MNRAS, 343, 75

Heavens A., Panter B., Jimenez R., Dunlop J., 2004, Nature, 428,625

Heckman T. M., Kauffmann G., Brinchmann J., Charlot S., Tremonti C., White S. D. M., 2004, ApJ, 613, 109

Hernquist L., 1990, ApJ, 356, 359

Hogg D. W., Blanton M. R., Brinchmann J., Eisenstein D. J., Schlegel D. J., Gunn J. E., McKay T. A., Rix H., Bahcall N. A., Brinkmann J., Meiksin A., 2004, ApJL, 601, L29

Hopkins P. F., Bundy K., Hernquist L., Ellis R. S., 2007, ApJ, 659, 976

Hopkins P. F., Cox T. J., Keres D., Hernquist L., 2007, ArXiv e-prints, 706

Hopkins P. F., Hernquist L., Cox T. J., Di Matteo T., Martini P., Robertson B., Springel V., 2005, ApJ, 630, 705

Im M., Faber S. M., Gebhardt K., Koo D. C., Phillips A. C., Schiavon R. P., Simard L., Willmer C. N. A., 2001, AJ, 122,750

Jimenez R., Panter B., Heavens A. F., Verde L., 2005, MNRAS, 356, 495

Juneau S., Glazebrook K., Crampton D., McCarthy P. J., Savaglio S., Abraham R., Carlberg R. G., Chen H.-W., Le Borgne 2005, ApJL, 619, L135 
Kauffmann G., Haehnelt M., 2000, MNRAS, 311, 576

Kauffmann G., Heckman T. M., White S. D. M., Charlot S., Tremonti C., Peng E. W., Seibert M., Brinkmann J., Nichol R. C., SubbaRao M., York D., 2003, MNRAS, 341, 54

Kennicutt R. C., 1983, ApJ, 272, 54

Kereš D., Katz N., Weinberg D. H., Davé R., 2005, MNRAS, 363, 2

Khochfar S., Ostriker J. P., 2007, ArXiv e-prints, 704

Kitzbichler M. G., White S. D. M., 2006, ArXiv Astrophysics e-prints

Kodama T., Yamada T., Akiyama M., Aoki K., Doi M., Furusawa H., Fuse T., Imanishi M., Ishida C., Iye M., Kajisawa M., 2004, MNRAS, 350, 1005

Kormendy J., Bender R., 1996, ApJL, 464, L119+

Lauer T. R., Gebhardt K., Faber S. M., Richstone D., Tremaine S., Kormendy J., Aller M. C., Bender R., Dressler A., Filippenko A. V., Green R., Ho L. C., 2007, ApJ, 664, 226

Lin H., Yee H. K. C., Carlberg R. G., Ellingson E., 1997, ApJ, 475, 494

Lin H., Yee H. K. C., Carlberg R. G., Morris S. L., Sawicki M., Patton D. R., Wirth G., Shepherd C. W., 1999, ApJ, 518,533

McCarthy I. G., Frenk C. S., Font A. S., Lacey C. G., Bower R. G., Mitchell N. L., Balogh M. L., Theuns T., 2007, ArXiv e-prints, 710

Neistein E., van den Bosch F. C., Dekel A., 2006, MNRAS, 372,933

Nelan J. E., Smith R. J., Hudson M. J., Wegner G. A., Lucey J. R., Moore S. A. W., Quinney S. J., Suntzeff N. B., 2005, ApJ, 632, 137

Nieto J.-L., Bender R., 1989, A\&A, 215, 266

Nieto J.-L., Bender R., Surma P., 1991, A\&A, 244, L37

Nipoti C., Binney J., 2007, ArXiv e-prints, 707

Noeske K. G., Faber S. M., Weiner B. J., Koo D. C., Primack J. R., Dekel A., Papovich C., Conselice C. J., Le Floc'h E., Rieke G. H., Coil A. L., Lotz J. M., Somerville R. S., Bundy K., 2007, ApJL, 660, L47

Omma H., Binney J., 2004, MNRAS, 350, L13

Pannella M., Hopp U., Saglia R. P., Bender R., Drory N., Salvato M., Gabasch A., Feulner G., 2006, ApJL, 639, L1 Panter B., Jimenez R., Heavens A. F., Charlot S., 2006, ArXiv Astrophysics e-prints

Rafferty D. A., McNamara B. R., Nulsen P. E. J., Wise M. W., 2006, ApJ, 652, 216

Rees M. J., Ostriker J. P., 1977, MNRAS, 179, 541

Rudnick G., Rix H.-W., Franx M., Labbé I., Blanton M., Daddi E., Förster Schreiber N. M., Moorwood A., Röttgering H., Trujillo I., van de Wel A., van der Werf P., van Dokkum P. G., van Starkenburg L., 2003, ApJ, 599, 847

Ruszkowski M., Brüggen M., Begelman M. C., 2004, ApJ, 615,675

Sandage A., 1986, A\&A, 161, 89

Schawinski K., Thomas D., Sarzi M., Maraston C., Kaviraj S., Joo S.-J., Yi S. K., Silk J., 2007, MNRAS, 382, 1415

Searle L., Sargent W. L. W., Bagnuolo W. G., 1973, ApJ, 179,427

Silk J., 1977, ApJ, 211, 638

Somerville R. S., Primack J. R., 1999, MNRAS, 310, 1087

Springel V., Di Matteo T., Hernquist L., 2005a, ApJL, 620,
L79

Springel V., Di Matteo T., Hernquist L., 2005b, MNRAS, 361,776

Steffen A. T., Barger A. J., Cowie L. L., Mushotzky R. F., Yang Y., 2003, ApJL, 596, L23

Strateva I., Ivezić Ž., Knapp G. R., Narayanan V. K., Strauss M. A., Gunn J. E., Lupton R. H., Schlegel D., Bahcall N. A., Brinkmann J., Brunner R. J., Budavári T., 2001, AJ, 122, 1861

Sutherland R. S., Dopita M. A., 1993, ApJS, 88, 253

Thomas D., Maraston C., Bender R., Mendes de Oliveira C., 2005, ApJ, 621, 673

Tinsley B. M., 1973, ApJ, 186, 35

Toomre A., Toomre J., 1972, ApJ, 178, 623

Tremblay B., Merritt D., 1996, AJ, 111, 2243

Ueda Y., Akiyama M., Ohta K., Miyaji T., 2003, Astronomische Nachrichten, 324, 36

van den Bosch F. C., 1998, ApJ, 507, 601

Voit G. M., Donahue M., 2005, ApJ, 634, 955

Weiner B. J., Phillips A. C., Faber S. M., Willmer C. N. A., Vogt N. P., Simard L., Gebhardt K., Im M., Koo D. C., Sarajedini V. L., Wu K. L., Forbes D. A., Gronwall C., Groth E. J., Illingworth G. D., Kron R. G., Rhodes J., Szalay A. S., Takamiya M., 2005, ApJ, 620, 595

White S. D. M., Rees M. J., 1978, MNRAS, 183, 341

\section{APPENDIX A: GALAXY MASS FUNCTIONS AT $Z \sim 0$ AND $Z \sim 1$}

The evolution of the mass functions of red and blue galaxies, whether increasing more strongly at low mass or at high mass, has been taken as a barometer of whether the red sequence forms primarily through downsizing or upsizing Bundy et al. 2005; Borch et al. 2006; Pannella et al. 2006; also see the discussion in Faber et al. 2007). In Section 7, we have shown how the evolution of the mass function of red galaxies is driven by a combination of star formation downsizing (due to quenching) and stellar mass assembly upsizing (due to merging). We have argued that the inherent difficulties in interpreting model mass functions, together with the large uncertainties that are still present in the models themselves, should suggest caution in using mass function data to infer up- or downsizing. In this Appendix, we consider the observational data on mass functions at $z \sim 0$ and $z \sim 1$.

The SDSS and 2MASS are the main data sets used to study the mass function of galaxies at $z \sim 0$ (e.g. Bell et al. 2003). Meanwhile, surveys that are increasingly deep (MUNICS, FDF, GOODS) are beginning to give us estimates for the mass function of galaxies at $z \sim 1$ (e.g. Bundy et al. 2006; Pannella et al.2006). Observational determinations of galaxy mass functions are affected by three sources of error, which we have enumerated in Section 7, and which we now discuss in greater detail.

The first is the steep slope of the mass function at the bright end. Therefore, even small random mass errors create large errors in the counts. The observed mass function at the bright end is not the true function but rather the true function smoothed by errors. Owing to the steepness of the bright end, this tends to falsely inflate the number of massive objects (the so-called Eddington effect; see also Kitzbichler \& White 2006 and Lauer et al. 2007). Random 
rms errors of only 0.2 dex in mass inflate observed counts by a full order of magnitude at $M_{\text {star }} \sim 10^{11.5} M_{\odot}$ (compare the solid and the dashed lines in Fig. A1). Model counts must therefore be smoothed to mimic this effect before comparing to data, but it is unclear whether errors get smaller or larger with redshift, and thus whether the evolutionary effect is positive or negative. Fig. A1 shows that we can make our model agree with one data set or the other simply by making different assumptions concerning the amplitude of the random measurement errors.

A second source that affects the photometry of the most massive galaxies is their having shallow light profiles that do not converge to a total magnitude. The magnitudes of very bright ellipticals are hard to measure on account of their extended envelopes, which may be growing with time due to galaxy-galaxy interactions. It is possible that many of them extend outside the photometric aperture used to measure their luminosities. Simply fitting such galaxies with de Vaucouleurs $R^{1 / 4}$ profiles is not appropriate because many profiles (at least locally) are even more extended than that (e.g., Graham et al. 2001). Total magnitude errors may therefore be larger for nearby galaxies than for distant ones. For example, the total luminosities of the brightest cluster galaxies of nearby Abell clusters can vary by many tenths of a magnitude (Gonzalez et al. 2005) depending on the profile fitting method used. Lauer et al. (2007) find errors of up to a magnitude in SDSS luminosities for such galaxies. These are very large errors when one considers that a change of only $0.2 \mathrm{mag}$ at $4 L_{*}$ translates to a change in number density of a factor of two in the Schechter function. Stellar masses have even larger errors because they depend on assumed evolution models for the fade in $M / L_{B}$, which introduce an additional uncertainty of a few tenths of a magnitude. While random errors are more likely to be important at high redshift, the problem deriving from the extended envelops of massive ellipticals is likely to worsen at low redshift, since a lot of the growth by dry merging occurs between $z \sim 1$ and $z \sim 0$ (Fig. 4). Therefore, the combination of these two sources of error is more likely to underestimate the evolution of the number of massive early type galaxies from $z \sim 1$ to $z \sim 0$.

Thirdly, before comparing mass functions from different authors, it is necessary to convert them first to the same assumed IMF. Bell et al. (2003) used a 'diet' Salpeter IMF, which corresponds to a Salpeter IMF truncated at low stellar masses Bell \& de Jong 2001). To convert the masses obtained Pannella et al. (2006) for a standard Salpeter IMF into masses that can be compared with the results by Bell et al. (2003), one must subtract $\sim 0.15$ dex from the masses determined by Pannella et al. (2006). Bundy et al. (2006) used a Chabrier IMF. The masses estimated with the Chabrier IMF are lower than those estimated with a Salpeter IMF by $\sim 0.25$ dex. Therefore, we must add $0.1 \mathrm{dex}$ to the masses determined by Bundy et al. (2006) to compare their mass function with that by Bell et al. (2003). After performing these corrections, we see that the apparent agreement between the figures in Bundy et al. (2006) and Pannella et al. (2006) is simply due to the fact that they used different IMFs. For reasons that we cannot easily interpret, with the same IMF, the galaxy mass function inferred by Bundy et al. (2006) contains many more massive galaxies at $z \sim 1$ than the mass function inferred by Pannella et al. (2006; Fig. A1). The data by Bundy et al. (2006) are consistent with no evolution at $M_{\text {star }} \gtrsim 10^{11} M_{\odot}$ in the redshift interval $0 \lesssim z \lesssim 1$. The data by Pannella et al. (2006) are consistent with growth in mass by $0.2-03$ dex at $M_{\text {star }} \gtrsim 10^{11} M_{\odot}$

In conclusion, it appears that the present state of mass functions at $z=0$ and $z=1$ is sufficiently confused to prevent firm conclusions. Problems are particularly severe at the high-mass end, where random errors seriously inflate counts at a given mass and questions persist about how to measure total light and mass. Fortunately, uncertainties are much smaller near $L_{*}$. Though the data are not perfect, the general trend is for red galaxies to increase in number with time near the knee of the mass function, by amounts that are in broad agreement with both luminosity function data (Faber et al. 2007) and with the predictions of our model. 

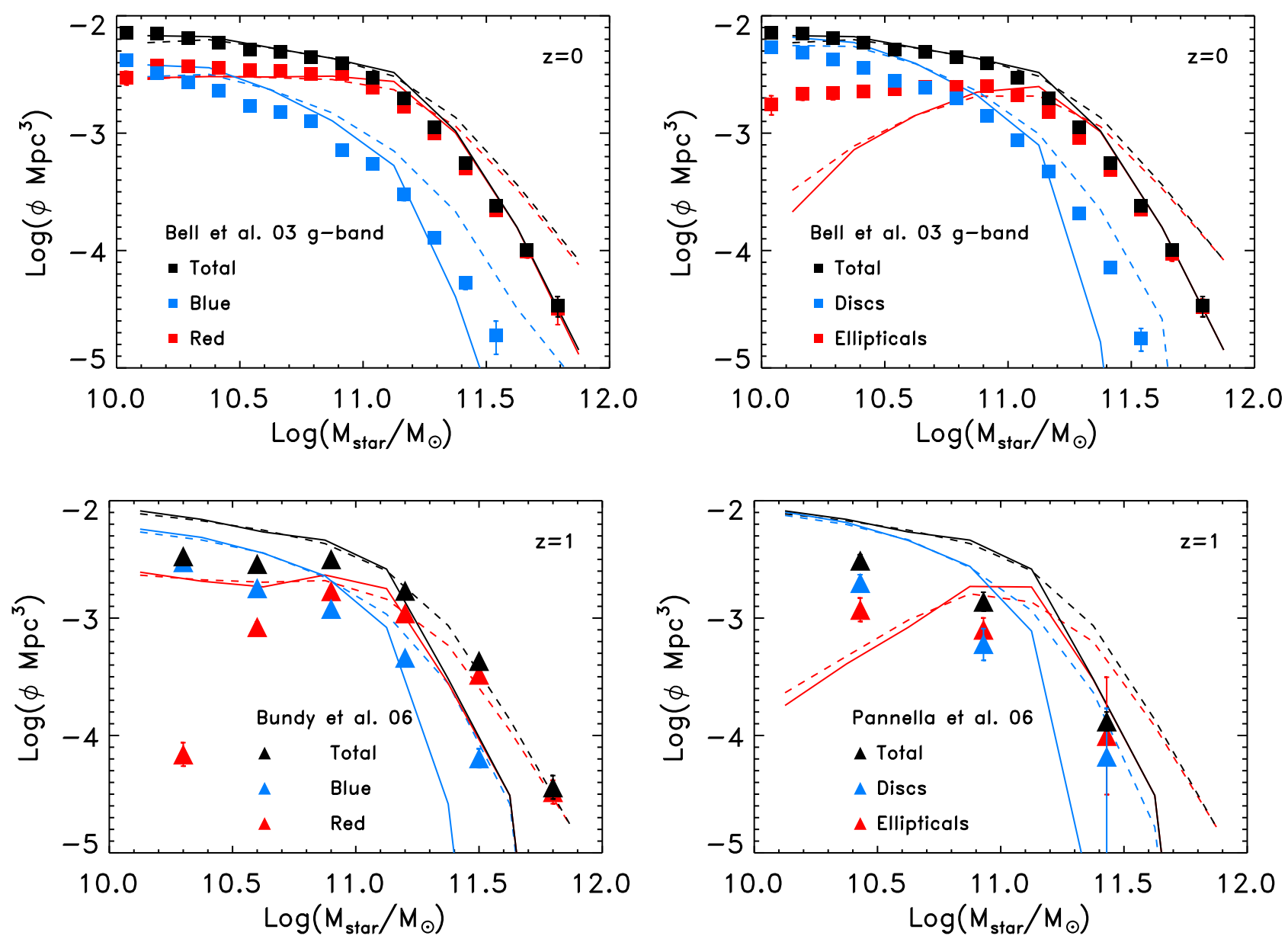

Figure A1. Mass functions at $z=0$ and $z=1$ for early type galaxies (red) and late type galaxies (blue) classified spectrally (left) and morphologically (right) The coloured and black points with error bars are the data. The lines show the predictions of our new model with star formation shutdown without (solid lines) and with (dashed lines) the inclusion of a 0.2 dex Gaussian random error. Note how the predictions of the model are completely altered at the massive end when such smoothing is included. 